\title{
Expert Arguments for Trends of Psychiatric Bed Numbers: A Systematic Review of Qualitative Data
}

\author{
Adrian P. Mundt ${ }^{1,2 *}$, Sabine Delhey Langerfeldt ${ }^{1}$, Enzo Rozas Serri ${ }^{1,3}$, \\ Mathias Siebenförcher ${ }^{4 *}$ and Stefan Priebe ${ }^{5}$
}

${ }^{1}$ Medical Faculty, Universidad Diego Portales, Santiago, Chile, ${ }^{2}$ Departamento de Neurología y Psiquiatría, Clínica Alemana de Santiago, Facultad de Medicina Clínica Alemana, Universidad del Desarrollo, Santiago, Chile, ${ }^{3}$ Department of Psychiatry and Mental Health, Hospital Clínico Universidad de Chile, Santiago, Chile, ${ }^{4}$ Department of Psychiatry and Psychotherapy Campus Mitte, Charité Universitätsmedizin Berlin, Berlin, Germany, ${ }^{5}$ Unit for Social and Community Psychiatry (WHO Collaborating Centre for Mental Health Service Development), Queen Mary University of London, London, United Kingdom

OPEN ACCESS

Edited by:

Anastasia Theodoridou, Psychiatric University Hospital Zurich, Switzerland

Reviewed by:

Ravi Philip Rajkumar, Jawaharlal Institute of Postgraduate Medical Education and Research (JIPMER), India

Siddharth Sarkar,

All India Institute of Medical

Sciences, India

*Correspondence:

Adrian P. Mund

adrian.mundt@mail.udp.cl

Mathias Siebenförcher mathias.siebenfoercher@charite.de

Specialty section:

This article was submitted to

Public Mental Health

a section of the journal

Frontiers in Psychiatry

Received: 21 July 2021 Accepted: 11 November 2021 Published: 24 December 2021

Citation:

Mundt AP, Delhey Langerfeldt S, Rozas Serri E, Siebenförcher $M$ and Priebe $S$ (2021) Expert Arguments for Trends of Psychiatric Bed Numbers: A Systematic Review of Qualitative Data.

Front. Psychiatry 12:745247.

doi: 10.3389/fpsyt.2021.745247
Introduction: Mental health policies have encouraged removals of psychiatric beds in many countries. It is under debate whether to continue those trends. We conducted a systematic review of expert arguments for trends of psychiatric bed numbers.

Methods: We searched seven electronic databases and screened 15,479 papers to identify expert opinions, arguments and recommendations for trends of psychiatric bed numbers, published until December 2020. Data were synthesized using thematic analysis and classified into arguments to maintain or increase numbers and to reduce numbers.

Results: One hundred six publications from 25 countries were included. The most common themes arguing for reductions of psychiatric bed numbers were inadequate use of inpatient care, better integration of care and better use of community care. Arguments to maintain or increase bed numbers included high demand of psychiatric beds, high occupancy rates, increasing admission rates, criminalization of mentally ill, lack of community care and inadequately short length of stay. Cost effectiveness and quality of care were used as arguments for increase or decrease.

Conclusions: The expert arguments presented here may guide and focus future debate on the required psychiatric bed numbers. The recommendations may help policymakers to define targets for psychiatric bed numbers. Arguments need careful local evaluation, especially when supporting opposite directions of trends in different contexts.

Keywords: psychiatric hospital beds, general hospital psychiatry, institutionalization, expert recommendation, consensus, inpatient, length of stay

\section{INTRODUCTION}

\section{Rationale}

Since the 1950's mental health services have undergone important transformations $(1,2)$. These included the development of community mental health care and the closure of psychiatric asylums and hospitals, which in most cases were built in the previous century. Reforms encouraged both reductions of psychiatric bed numbers and length of stay for psychiatric hospitalization, 
and promoted long-stay housing facilities in the community (3). At present, the reforms are still ongoing in many places. Substantial parts of inpatient psychiatric services have been removed and services have shifted toward community care (46). Reforms have not established a minimum or optimal number of psychiatric beds in order to assure balanced mental health systems, and it remains under debate whether to continue to remove psychiatric beds $(7-10)$. Furthermore, research is still scarce on how many beds have actually been removed in the context of reforms and how these changes relate to other types of institutionalization (8).

The rates of psychiatric beds differ between countries and geographical regions (11), especially between different income groups (12). According to the WHO Mental Health Atlas (2017), the median number of psychiatric beds per 100,000 population is around 50 in high income countries (HIC), opposed to a rate of 7 in low and middle-income countries (LMIC). Residential care beds are almost inexistent in LMIC, whereas a median of 23 residential care beds per 100,000 population were reported for HIC (11).

The definition of psychiatric beds used by the Mental Health Atlas Project (11) incorporates short-stay and long-stay beds in psychiatric hospitals, beds in general hospital psychiatric units (GHPU), inpatient psychiatric services based in community settings and forensic inpatient units. This includes public and private facilities, psychiatric beds only for children and adolescents and other specific groups such as older adults. The definition excludes beds, which are exclusively used for the treatment of individuals with intellectual disability or substance use disorders, as well as facilities that exclusively provide rehabilitation and recovery services. The exact definition of beds in long-stay facilities causes difficulties $(10,13)$. The concept of psychiatric beds has undergone substantial changes over time $(14,15)$. This global variety of definitions limits international comparisons that aim to develop and optimize services $(10,16-$ 18). A review assessing mental health plans of five Englishspeaking HIC showed that even in regions with the same official language, recommendations used variable nomenclature and mostly promoted a mix of inpatient and community services (16). It showed the need to define core mental health service components including specific resource targets in order to deliver more strategic clinical care.

Three different approaches have been proposed for estimating the required number of psychiatric beds (18). First, an empirical population health approach that estimates or calculates current and future psychiatric bed requirements for a specific catchment area based on epidemiological data considering current provision and quality of care (19). Secondly, expert consensus has been conducted in developed countries (20-22). Thirdly, a normative approach that assumes that different catchment areas with similar mental health and demographic profiles may require a similar number of psychiatric beds, so a well-functioning mental health system can be used as a model for other similar areas (18, 19).

We conducted a systematic review on expert opinions, arguments and recommendations for trends of psychiatric bed numbers.

\section{METHODS}

\section{Database Searches}

The following seven databases were searched from their inception until December 27, 2020: PubMed, Embase Classic and Embase, PsycINFO and PsycIndex, Open Gray, Google Scholar, Global Health EBSCO and Proquest Dissertations. The search term used was "psychiatric AND hospital* AND bed*" with no filters based on study types. We did not use any language restrictions. Since Google Scholar produces very high numbers of hits $(>500,000)$ and sorts them by relevance, the search was limited to the first 561 hits. We restricted searches in PubMed, PsycINFO and PsycIndex to title and abstract. This helped us to assure that the psychiatric beds were a central topic of the articles rather than tangentially discussed. References and citations of articles retained in this study were reviewed for additional unidentified studies.

\section{Eligibility Criteria}

We included studies that presented arguments, opinions, recommendations and suggestions for trends of psychiatric bed numbers. We excluded studies that only reported specific numbers of psychiatric beds. Studies providing purely numerical recommendations were excluded from this paper. Publications referring to beds or places in other mental health facilities such as residential facilities or day hospitals were also excluded.

\section{Data Collection Process}

Literature screening was conducted by SD, Dr. ERS, and Dr. MS. Data were extracted independently by SD, Dr. ERS, and Dr. MS.

\section{Data Extraction}

The following variables were extracted: year of publication, whether the expert recommendation referred to a local, national, regional or global area, the income group of the country for which the recommendation was made according to the World Bank classification (12), author's hypothesis or argument for the proposed change and recommendations. Multiple arguments were extracted, if present in the same publications.

\section{Data Analysis}

The respective recommendations were a priori classified whether they dealt with acute care and short-stay on the one hand and long-stay on the other hand. When short- and mediumstay beds were reported in an aggregated format, they were classified as short-stay and when medium to long-stay beds were reported in an aggregated way, they were classified as long-stay. Furthermore, recommendations were grouped by suggestion to either reduce and maintain or increase psychiatric bed numbers. The arguments were analyzed using a thematic analysis approach with six steps (23), which is a qualitative analytic method used to identify, analyze and report codes, subthemes and themes for qualitative data. We used a theoretical rather than inductive thematic analyses approach (23), since the themes were sorted into the overarching a priori defined groups "reduce" or "increase- or maintain" psychiatric bed numbers. The themes were identified on a latent rather than semantic level (23). Findings from different publications were integrated 
in order to achieve an interpretation of the arguments that resulted in a higher level of scientific evidence, consistent with meta-synthesis (24). After familiarization with the texts, an open coding was developed and initial codes were determined. Then initial codes were grouped into different categories according to their similarities. In the third step, these categories were organized into themes. This implied the combination of codes into wider and overarching themes that adequately reflect the extracted information. Three authors subsequently examined this classification reorganizing the data and recoding the categories in a reiterative process between researchers and ensuring that categories were understood in the same way by all researchers. In the fifth step, a comprehensive analysis of the different themes was conducted ensuring that each one contributed to a better understanding of the data. For each category, a summary of the main ideas was derived from all included quotes. In the sixth and final step, the report was written allowing for a detailed description of the results. To ensure robustness, quotes were included in the description of the results to illustrate the descriptions.

The number of quoted recommendations per country and category were analyzed.

Critical appraisal tools typically aim to assess the quality of a study in particular. As quoted recommendations and expert arguments were not necessarily identical to the main objective of the respective studies, the methodology and overall quality of the publication were not suitable to assess the quality of the opinion. Thus, by consensus between the authors, we did not rank or differentiate the quality of the included recommendations or arguments. Nonetheless, in order to ensure validity of the results several quality criteria were included. First, triangulation was implemented meaning three complementary researchers from different backgrounds (psychology and psychiatry) participated in the data analysis. Secondly, theoretical validation was used to compare results with the scientific literature. Lastly, an iterative process was conducted. When a new code was added, the codification was read again in order to ensure that the initial classification was accurate and the extracted data were complete.

\section{RESULTS}

We followed the guidelines for Preferred Reporting Items of Systematic Reviews (PRISMA). The PRISMA flow chart is shown in Figure 1.

Expert arguments for trends of psychiatric bed numbers were reported in 106 publications (Table 1). Fifty studies made recommendations for short-stay beds, twelve for long-stay beds and 44 did not specify the length of stay. The studies were published between 1960 and 2020 from 25 different countries, including one study from Canada and Australia (10), one for global recommendations (WHO) (58) and one for the Eastern Mediterranean Region (EMR) (71). Ninety-two publications were from high-income countries (HIC), most from the United Kingdom and the United States. Fourteen studies referred to LMIC including Bhutan (68), Brazil $(35,53,104)$, China (94), Ghana (74, 114), Moldova (88), Malawi (76), South Africa

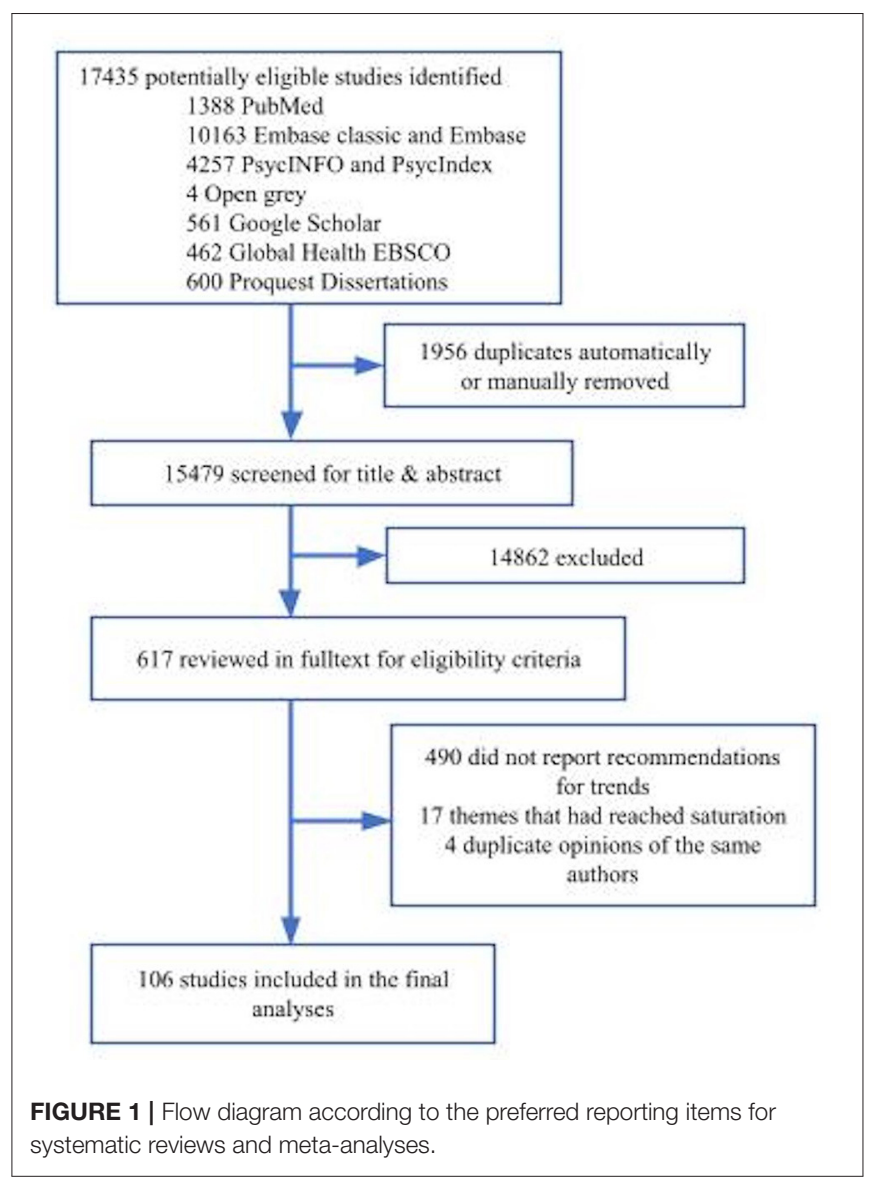

(55, 62), Uganda (103) and two publications referred to South America $(8,105)$. In 59 studies, recommendations were based on local or national epidemiological and demographical data. Recommendations based on expert consensus or opinion were found in 38 publications. Eleven recommendations were in line with a normative approach using institutional or governmental guidelines $(14,36,53,58,79,81,88,98,103,106)$. Two studies combined approaches $(14,88)$. Most expert arguments came from HIC (31) and only few (10) from LMIC (Table 2).

\section{Extracted Themes}

We distinguished two main categories: (a) arguments to reduce psychiatric bed numbers, and (b) arguments to maintain or increase psychiatric bed numbers. In both categories, we identified themes and subthemes supporting each trend (Annex 1). We identified 36 quotes arguing for a decrease and 123 quotes supporting to increase or maintain psychiatric bed numbers.

Illustrative verbatim are given in Table 3. A thematic map was built in order to show the main themes that emerged from the data (Figures 2, 3), which are described in further detail below.

\section{Expert Arguments to Reduce Psychiatric Bed Numbers}

Ten themes emerged as arguments supporting a trend to reduce psychiatric bed numbers. 
TABLE 1 | Publications reporting recommendations and arguments for trends of psychiatric bed numbers sorted by length of stay.

\begin{tabular}{|c|c|c|c|c|c|c|c|c|c|}
\hline References & $\begin{array}{l}\text { Year of } \\
\text { publication }\end{array}$ & Country & $\begin{array}{l}\text { Income } \\
\text { group }\end{array}$ & $\begin{array}{l}\text { International, } \\
\text { national or } \\
\text { local scope } \\
\text { [catchment } \\
\text { area or } \\
\text { jurisdiction } \\
\text { when local] }\end{array}$ & $\begin{array}{l}\text { Type of } \\
\text { publication }\end{array}$ & $\begin{array}{l}\text { Length of } \\
\text { stay }\end{array}$ & $\begin{array}{l}\text { Approach to support } \\
\text { recommendation }\end{array}$ & $\begin{array}{l}\text { Suggested } \\
\text { trend of } \\
\text { psychiatric } \\
\text { bed } \\
\text { numbers }\end{array}$ & Argument for change per theme \\
\hline \multicolumn{10}{|c|}{ Acute, short-stay and General Hospital psychiatric beds } \\
\hline $\begin{array}{l}\text { Allison and Bastiampillai } \\
\text { (25) }\end{array}$ & 2015 & Australia & $\mathrm{HI}$ & $\begin{array}{l}\text { Local [South } \\
\text { Australia] }\end{array}$ & Perspective & Acute & Expert opinion & Increase & $\begin{array}{l}\text { Overcrowding and long waiting times in ED and early } \\
\text { readmission }\end{array}$ \\
\hline Baia Medeiros et al. (26) & 2019 & Canada & $\mathrm{HI}$ & Local [Toronto] & Original research & Acute (ED) & Original forecast & Increase & $\begin{array}{l}\text { Hardships for patients and families, compromised safety } \\
\text { and occurrence of serious incidents }\end{array}$ \\
\hline Bastiampillai et al. (5) & 2010 & Australia & $\mathrm{HI}$ & $\begin{array}{l}\text { Local } \\
\text { [Adelaide] }\end{array}$ & Original research & Acute & Original estimate & Decrease & $\begin{array}{l}\text { New care pathways and better integration of emergency } \\
\text { departments, inpatient and outpatient services allow for } \\
\text { further psychiatric bed removals }\end{array}$ \\
\hline Bloom (27) & 2015 & US & $\mathrm{HI}$ & $\begin{array}{l}\text { Local } \\
\text { [Washington } \\
\text { state] }\end{array}$ & $\begin{array}{l}\text { Analysis \& } \\
\text { commentary }\end{array}$ & Acute (ED) & Expert opinion & Increase & Overcrowding and long waiting times in ED \\
\hline Claudius (28) & 2019 & US & $\mathrm{HI}$ & National & Original research & $\begin{array}{l}\text { Acute } \\
\text { (pediatric, ED) }\end{array}$ & Original estimate & $\begin{array}{l}\text { Do not } \\
\text { increase }\end{array}$ & $\begin{array}{l}\text { Bed reductions do not affect the quality of care in the } \\
\text { system as a whole and has not shown negative effects }\end{array}$ \\
\hline Davie (29) & 2019 & Australia & $\mathrm{HI}$ & National & Letter & Acute & Expert opinion & Increase & $\begin{array}{l}\text { Increasing suicide rates, insufficient and ineffective } \\
\text { community services }\end{array}$ \\
\hline Dhillon (30) & 2015 & Australia & $\mathrm{HI}$ & $\begin{array}{l}\text { Local [South } \\
\text { Australia] }\end{array}$ & Correspondence & Acute & Expert opinion & Increase & Overcrowding and long waiting times in ED \\
\hline Duthie (31) & 2001 & UK & $\mathrm{HI}$ & Local [Wales] & Correspondence & $\begin{array}{l}\text { Acute } \\
\text { (pediatric, ED) }\end{array}$ & Expert opinion & Increase & $\begin{array}{l}\text { Lack of specialized psychiatric beds for children and } \\
\text { adolescents }\end{array}$ \\
\hline Early and Nicholas (32) & 1971 & UK & $\mathrm{HI}$ & Local [Bristol] & Original research & GHPU & Original estimate & $\begin{array}{l}\text { Not do } \\
\text { reduce }\end{array}$ & $\begin{array}{l}\text { Increasing admission rates and waiting times, } \\
\text { inappropriate admission due to lack of alternative care }\end{array}$ \\
\hline Early and Nicholas (33) & 1977 & UK & $\mathrm{HI}$ & Local [Bristol] & Original research & GHPU & Original estimate & Decrease & Inappropriately long psychiatric inpatient care \\
\hline Elpers and Crowell (34) & 1982 & US & $\mathrm{HI}$ & $\begin{array}{l}\text { Global } \\
\text { [developed } \\
\text { countries] }\end{array}$ & Overview & Acute & Expert opinion & $\begin{array}{l}\text { Not to } \\
\text { eliminate }\end{array}$ & Insufficient and ineffective community service \\
\hline $\begin{array}{l}\text { Fagundes-Junior et al. } \\
\text { (35) }\end{array}$ & 2016 & Brazil & UMI & $\begin{array}{l}\text { Local [Rio de } \\
\text { Janeiro] }\end{array}$ & Original research & GHPU & Original estimate & $\begin{array}{l}\text { Stop bed } \\
\text { reductions }\end{array}$ & Insufficient and ineffective community services \\
\hline Flannigan et al. (36) & 1994 & UK & $\mathrm{HI}$ & Local [London] & Original research & Acute & Guidelines (normative) & Increase & $\begin{array}{l}\text { Discharge to homelessness and shelters, increasing } \\
\text { admission rates and waiting times }\end{array}$ \\
\hline Ford, et al. (37) & 2001 & UK & $\mathrm{HI}$ & $\begin{array}{l}\text { Local [North } \\
\text { Birmingham] }\end{array}$ & Original research & Acute & Original estimate & Reduce & Lower cost of home treatment and outpatient care \\
\hline Friebel et al. (38) & 2019 & UK & $\mathrm{HI}$ & $\begin{array}{l}\text { National } \\
\text { [England] }\end{array}$ & Original research & Acute & Original estimate & Increase & Short length of stay and premature discharge \\
\hline Fulop et al. (39) & 1996 & UK & $\mathrm{HI}$ & $\begin{array}{l}\text { Local [North } \\
\text { and South } \\
\text { Thames] }\end{array}$ & Original research & Acute & Original estimate & $\begin{array}{l}\text { Not to } \\
\text { increase }\end{array}$ & $\begin{array}{l}\text { Inappropriately long psychiatric inpatient care, new care } \\
\text { pathways and better integration of emergency } \\
\text { departments, inpatient and outpatient services allow for } \\
\text { further psychiatric bed removals }\end{array}$ \\
\hline
\end{tabular}




\begin{tabular}{|c|c|c|c|c|c|c|c|c|c|}
\hline References & $\begin{array}{l}\text { Year of } \\
\text { publication }\end{array}$ & Country & $\begin{array}{l}\text { Income } \\
\text { group }\end{array}$ & $\begin{array}{l}\text { International, } \\
\text { national or } \\
\text { local scope } \\
\text { [catchment } \\
\text { area or } \\
\text { jurisdiction } \\
\text { when local] }\end{array}$ & $\begin{array}{l}\text { Type of } \\
\text { publication }\end{array}$ & $\begin{array}{l}\text { Length of } \\
\text { stay }\end{array}$ & $\begin{array}{l}\text { Approach to support } \\
\text { recommendation }\end{array}$ & $\begin{array}{l}\text { Suggested } \\
\text { trend of } \\
\text { psychiatric } \\
\text { bed } \\
\text { numbers }\end{array}$ & Argument for change per theme \\
\hline Harris (40) & 1975 & US & $\mathrm{HI}$ & $\begin{array}{l}\text { Local [New } \\
\text { York State] }\end{array}$ & Original research & GHPU & Original estimate & Reduce & $\begin{array}{l}\text { Lower cost of home treatment and outpatient care, } \\
\text { inappropriately long psychiatric inpatient care }\end{array}$ \\
\hline Hatta et al. (41) & 2010 & Japan & $\mathrm{HI}$ & Local [Tokyo] & Original research & GHPU & Original estimate & Increase & High occupancy rates and overcrowding \\
\hline Jones (42) & 2013 & UK & $\mathrm{HI}$ & Global & Original research & Acute & Original estimate & Increase & $\begin{array}{l}\text { Implementation of community care complements, but } \\
\text { does not replace inpatient care }\end{array}$ \\
\hline Kalucy et al. (43) & 2005 & Australia & $\mathrm{HI}$ & $\begin{array}{l}\text { Local } \\
\text { [Adelaide] }\end{array}$ & Original research & Acute (ED) & Original estimate & Increase & $\begin{array}{l}\text { Overcrowding and long waiting times in emergency } \\
\text { departments }\end{array}$ \\
\hline Kelly (44) & 1998 & Ireland & $\mathrm{HI}$ & $\begin{array}{l}\text { Local } \\
\text { [Northern } \\
\text { Ireland] }\end{array}$ & Original research & Acute & Original estimate & Increase & High occupancy rates and overcrowding \\
\hline Keown et al. (45) & 2007 & UK & $\mathrm{HI}$ & $\begin{array}{l}\text { Local } \\
\text { [Newcastle } \\
\text { and North } \\
\text { Tyneside] }\end{array}$ & Original research & Acute & Original estimate & Increase & $\begin{array}{l}\text { Financial pressure on the mental health system has } \\
\text { resulted in too many bed removals and underfunded } \\
\text { inpatient care systems }\end{array}$ \\
\hline La et al. (46) & 2016 & US & $\mathrm{HI}$ & $\begin{array}{l}\text { Local [North } \\
\text { Carolina] }\end{array}$ & Original research & Acute & Original estimate & Increase & Increasing admission rates and waiting times \\
\hline $\begin{array}{l}\text { Lamb and } \\
\text { Weinberger (47) }\end{array}$ & 2011 & US & $\mathrm{HI}$ & National & $\begin{array}{l}\text { Analysis \& } \\
\text { commentary }\end{array}$ & Acute & Expert opinion & Increase & $\begin{array}{l}\text { Increasing detention rates due to lack of adequate and } \\
\text { timely mental health treatments of persons with severe } \\
\text { mental illnesses (and comorbid substance use disorders) }\end{array}$ \\
\hline Laugharne et al. (48) & 2016 & UK & $\mathrm{HI}$ & $\begin{array}{l}\text { Local } \\
\text { [Cornwall] }\end{array}$ & Original research & Acute & Original estimate & Decrease & $\begin{array}{l}\text { Inappropriately long psychiatric inpatient care, inpatient } \\
\text { services are restrictive environments }\end{array}$ \\
\hline Lee et al. (49) & 2016 & Hong Kong & $\mathrm{HI}$ & National & Letter & Acute & Expert opinion & Increase & $\begin{array}{l}\text { Financial pressure on the mental health system has } \\
\text { resulted in too many bed removals and underfunded } \\
\text { inpatient care systems, high occupancy rates and } \\
\text { overcrowding }\end{array}$ \\
\hline Lelliott (50) & 1996 & UK & $\mathrm{HI}$ & $\begin{array}{l}\text { National } \\
\text { [England] }\end{array}$ & Original research & $\begin{array}{l}\text { Short-stay } \\
\text { (admission) }\end{array}$ & Expert opinion & Increase & $\begin{array}{l}\text { Increasing admission rates and waiting times, limited } \\
\text { post-discharge support in the community }\end{array}$ \\
\hline Lelliott (51) & 2006 & UK & $\mathrm{HI}$ & $\begin{array}{l}\text { National } \\
\text { [England] }\end{array}$ & Short report & Acute & Expert opinion & Increase & $\begin{array}{l}\text { Lack of specialized psychiatric beds for children and } \\
\text { adolescents, hardships for patients and families, } \\
\text { compromised safety and occurrence of serious incidents }\end{array}$ \\
\hline Lippert et al. (52) & 2016 & US & $\mathrm{HI}$ & National & Original research & Acute (ED) & Original estimate & Increase & $\begin{array}{l}\text { Overcrowding and long waiting times in emergency } \\
\text { departments, insufficient and ineffective community } \\
\text { services }\end{array}$ \\
\hline Loch et al. (53) & 2016 & Brazil & UMI & $\begin{array}{l}\text { Global [South } \\
\text { America] }\end{array}$ & Review & GHPU & Guidelines (normative) & Increase & Insufficient and ineffective community services \\
\hline
\end{tabular}




\begin{tabular}{|c|c|c|c|c|c|c|c|c|c|}
\hline References & $\begin{array}{l}\text { Year of } \\
\text { publication }\end{array}$ & Country & $\begin{array}{l}\text { Income } \\
\text { group }\end{array}$ & $\begin{array}{l}\text { International, } \\
\text { national or } \\
\text { local scope } \\
\text { [catchment } \\
\text { area or } \\
\text { jurisdiction } \\
\text { when local] }\end{array}$ & $\begin{array}{l}\text { Type of } \\
\text { publication }\end{array}$ & $\begin{array}{l}\text { Length of } \\
\text { stay }\end{array}$ & $\begin{array}{l}\text { Approach to support } \\
\text { recommendation }\end{array}$ & $\begin{array}{l}\text { Suggested } \\
\text { trend of } \\
\text { psychiatric } \\
\text { bed } \\
\text { numbers }\end{array}$ & Argument for change per theme \\
\hline Long (54) & 2015 & Australia & $\mathrm{HI}$ & Local & Correspondence & Acute & Expert opinion & Increase & Increasing admission rates and waiting times \\
\hline Lund and Flisher (55) & 2006 & South Africa & UMI & National & Original research & Acute & Original estimate & Increase & $\begin{array}{l}\text { Need for the development of integrated health care } \\
\text { systems with decentralized inpatient care capacities }\end{array}$ \\
\hline MacDonald et al. (56) & 1999 & New Zealand & $\mathrm{HI}$ & $\begin{array}{l}\text { Local } \\
\text { (Wellington) }\end{array}$ & Original research & Acute & Original estimate & Increase & High occupancy rates and overcrowding \\
\hline Malcolm (57) & 1989 & New Zealand & $\mathrm{HI}$ & National & Original research & $\begin{array}{l}\text { Short-stay } \\
\text { (admission) }\end{array}$ & Original estimate & Decrease & $\begin{array}{l}\text { Lower cost of home treatment and outpatient care, } \\
\text { inpatient psychiatric bed capacity and availability } \\
\text { generates utilization and coercive treatments }\end{array}$ \\
\hline Morris et al. (58) & 2012 & Global (WHO) & $H I \& L M l$ & $\begin{array}{l}\text { Global (184 } \\
\text { countries) }\end{array}$ & Original research & GHPU & Guidelines (normative) & $\begin{array}{l}\text { Balance bed } \\
\text { reduction } \\
\text { with increase } \\
\text { of community } \\
\text { care }\end{array}$ & $\begin{array}{l}\text { Sub-groups of people with severe mental illnesses Are } \\
\text { still in need of psychiatric inpatient beds }\end{array}$ \\
\hline Munk-Jorgensen (59) & 1999 & Denmark & $\mathrm{HI}$ & National & Original research & Acute & Expert opinion & $\begin{array}{l}\text { Do not } \\
\text { reduce }\end{array}$ & $\begin{array}{l}\text { High occupancy rates and overcrowding, increasing } \\
\text { admission rates and waiting times, increasing suicide } \\
\text { rates criminalization of mentally ill }\end{array}$ \\
\hline Nicks and Manthey (60) & 2012 & US & $\mathrm{HI}$ & Local (61) & Original research & Acute (ED) & Original estimate & Increase & $\begin{array}{l}\text { Overcrowding and long waiting times in emergency } \\
\text { departments }\end{array}$ \\
\hline Niehaus et al. (62) & 2008 & South Africa & UMI & $\begin{array}{l}\text { Local [Western } \\
\text { Cape } \\
\text { Province] }\end{array}$ & Original research & Acute & Original estimate & Increase & Early readmission rates \\
\hline Nordstrom et al. (63) & 2019 & US & $\mathrm{HI}$ & National & Original research & Acute (ED) & Expert opinion & Increase & $\begin{array}{l}\text { Financial disincentives and unfair reimbursement practice } \\
\text { have led to lower numbers of psychiatric beds than } \\
\text { actually needed }\end{array}$ \\
\hline O'Doherty (64) & 1998 & Ireland & $\mathrm{HI}$ & Local [NR] & Original research & Acute & Original estimate & Reduce & $\begin{array}{l}\text { Implementation of day hospital services and home } \\
\text { treatment teams allow for greater concentration of } \\
\text { inpatient resources on most severely ill patients, leading } \\
\text { to cost savings }\end{array}$ \\
\hline $\begin{array}{l}\text { O'Reilly and Chamberlaine } \\
\text { (65) }\end{array}$ & 2000 & Canada & $\mathrm{HI}$ & National & Letter & Acute & Expert opinion & $\begin{array}{l}\text { Do not } \\
\text { reduce }\end{array}$ & $\begin{array}{l}\text { Increasing admission rates and waiting times, hardships } \\
\text { for patients and families, compromised safety and } \\
\text { occurrence of serious incidents }\end{array}$ \\
\hline O'Neil et al. (66) & 2016 & US & $\mathrm{HI}$ & $\begin{array}{l}\text { Local } \\
\text { [Rochester, } \\
\text { Minnesota] }\end{array}$ & Original research & Acute (ED) & Original estimate & Increase & $\begin{array}{l}\text { Overcrowding and long waiting times in emergency } \\
\text { departments, risk of transfer outside patients' local } \\
\text { community for care, hardships for patients and families, } \\
\text { compromised safety and occurrence of serious incidents }\end{array}$ \\
\hline Parker et al. (67) & 2015 & Australia & $\mathrm{HI}$ & National & Correspondence & Acute & Expert opinion & Increase & $\begin{array}{l}\text { Implementation of community care complements, but } \\
\text { does not replace inpatient care }\end{array}$ \\
\hline
\end{tabular}




\begin{tabular}{|c|c|c|c|c|c|c|c|c|c|}
\hline References & $\begin{array}{l}\text { Year of } \\
\text { publication }\end{array}$ & Country & $\begin{array}{l}\text { Income } \\
\text { group }\end{array}$ & $\begin{array}{l}\text { International, } \\
\text { national or } \\
\text { local scope } \\
\text { [catchment } \\
\text { area or } \\
\text { jurisdiction } \\
\text { when local] }\end{array}$ & $\begin{array}{l}\text { Type of } \\
\text { publication }\end{array}$ & $\begin{array}{l}\text { Length of } \\
\text { stay }\end{array}$ & $\begin{array}{l}\text { Approach to support } \\
\text { recommendation }\end{array}$ & $\begin{array}{l}\text { Suggested } \\
\text { trend of } \\
\text { psychiatric } \\
\text { bed } \\
\text { numbers }\end{array}$ & Argument for change per theme \\
\hline Pelzang (68) & 2012 & Bhutan & LMI & $\begin{array}{l}\text { Local } \\
\text { [Thimphu] }\end{array}$ & Original research & $\begin{array}{l}\text { Short-stay } \\
\text { (admission) }\end{array}$ & Original estimate & Increase & Increasing admission rates and waiting times \\
\hline Powell et al. (69) & 1995 & UK & $\mathrm{HI}$ & $\begin{array}{l}\text { Local } \\
\text { [Greater } \\
\text { London] }\end{array}$ & Original research & $\begin{array}{l}\text { Short-stay } \\
\text { (admission) }\end{array}$ & Original estimate & Increase & High occupancy rates and overcrowding \\
\hline Prins (70) & 2011 & US & $\mathrm{HI}$ & National & Short report & Acute & Expert opinion & Increase & $\begin{array}{l}\text { Sub-groups of people with severe mental illnesses are } \\
\text { still in need of psychiatric inpatient beds, criminalization } \\
\text { of mentally ill }\end{array}$ \\
\hline Saraceno et al. (71) & 2015 & EMR & & $\begin{array}{l}\text { Regional } \\
\text { [EMR] }\end{array}$ & Original research & GHPU & Guidelines (normative) & Reduce & $\begin{array}{l}\text { Reduced number of long-stay patients allows for further } \\
\text { bed removals }\end{array}$ \\
\hline Shumway et al. (6) & 2012 & US & $\mathrm{HI}$ & $\begin{array}{l}\text { Local [San } \\
\text { Francisco] }\end{array}$ & Original research & Acute & Original estimate & Reduce & $\begin{array}{l}\text { Bed reductions do not affect the quality of care in the } \\
\text { system as a whole and has not shown negative effects }\end{array}$ \\
\hline Thomas (72) & 2003 & US & $\mathrm{HI}$ & National & Editorial & $\begin{array}{l}\text { Acute } \\
\text { (pediatric, ED) }\end{array}$ & Expert opinion & Increase & $\begin{array}{l}\text { Short length of stay and premature discharge, lack of } \\
\text { specialized psychiatric beds for children and adolescents }\end{array}$ \\
\hline Tyrer et al. (73) & 2017 & UK & $\mathrm{HI}$ & National & Correspondence & Acute & Expert opinion & Increase & $\begin{array}{l}\text { Short length of stay and premature discharge, risk of } \\
\text { transfer outside patients' local community for care, } \\
\text { hardships for patients and families, compromised safety } \\
\text { and occurrence of serious incidents, increase in } \\
\text { involuntary admissions due to lack of timely voluntary } \\
\text { admission at an earlier stage of illness, implementation of } \\
\text { community care complements, but does not replace } \\
\text { inpatient care }\end{array}$ \\
\hline \multicolumn{10}{|c|}{ Medium - and long-stay } \\
\hline Akpalu et al. (74) & 2010 & Ghana & LI & National & Original research & Long-stay & Expert consensus & Increase & High occupancy \\
\hline Allison et al. (75) & 2018 & Australia & $\mathrm{HI}$ & Local [Victoria] & Commentary & Long-stay & Expert opinion & Increase & $\begin{array}{l}\text { Severe emotional and physical harm to patients, families } \\
\text { and communities }\end{array}$ \\
\hline Barnett et al. (76) & 2019 & Malawi & $\mathrm{LI}$ & $\begin{array}{l}\text { Local } \\
\text { [Lilongwe] }\end{array}$ & Original research & Long-stay & Original estimate & Increase & $\begin{array}{l}\text { Need for the development of integrated health care } \\
\text { systems with decentralized inpatient care capacities }\end{array}$ \\
\hline Giel (77) & 1986 & Netherlands & $\mathrm{HI}$ & National & Original research & Long-stay & Original estimate & $\begin{array}{l}\text { Not to } \\
\text { reduce }\end{array}$ & $\begin{array}{l}\text { Sub-groups of people with severe mental illnesses are } \\
\text { still in need of psychiatric inpatient beds }\end{array}$ \\
\hline Hailey (78) & 1971 & UK & $\mathrm{HI}$ & $\begin{array}{l}\text { Local } \\
\text { [Camberwell, } \\
\text { England] }\end{array}$ & Original research & Long-stay & Original forecast & Reduce & $\begin{array}{l}\text { Reduced number of long-stay patients allows for further } \\
\text { bed removals }\end{array}$ \\
\hline Holloway et al. (79) & 1999 & UK & $\mathrm{HI}$ & $\begin{array}{l}\text { Local [East } \\
\text { Lambeth and } \\
\text { South } \\
\text { Southwark, } \\
\text { London] }\end{array}$ & Original research & Long-stay & Guidelines (normative) & Decrease & $\begin{array}{l}\text { Reduced number of long-stay patients allows for further } \\
\text { bed removals, bed reductions lead to better use of } \\
\text { existing community care }\end{array}$ \\
\hline Kim (80) & 2017 & Korea & $\mathrm{HI}$ & National & Commentary & Long-stay & Expert opinion & Decrease & $\begin{array}{l}\text { Follow global trends of psychiatric bed reductions in } \\
\text { most of the developed countries }\end{array}$ \\
\hline
\end{tabular}

Barnett et al. (76)

Giel (77)

Hailey (78)

2017 


\begin{tabular}{|c|c|c|c|c|c|c|c|c|c|}
\hline References & $\begin{array}{l}\text { Year of } \\
\text { publication }\end{array}$ & Country & $\begin{array}{l}\text { Income } \\
\text { group }\end{array}$ & $\begin{array}{l}\text { International, } \\
\text { national or } \\
\text { local scope } \\
\text { [catchment } \\
\text { area or } \\
\text { jurisdiction } \\
\text { when local] }\end{array}$ & $\begin{array}{l}\text { Type of } \\
\text { publication }\end{array}$ & $\begin{array}{l}\text { Length of } \\
\text { stay }\end{array}$ & $\begin{array}{l}\text { Approach to support } \\
\text { recommendation }\end{array}$ & $\begin{array}{l}\text { Suggested } \\
\text { trend of } \\
\text { psychiatric } \\
\text { bed } \\
\text { numbers }\end{array}$ & Argument for change per theme \\
\hline Lelliott and Wing (14) & 1994 & UK & $\mathrm{HI}$ & $\begin{array}{l}\text { Global } \\
\text { [England \& } \\
\text { Wales, } \\
\text { Scotland and } \\
\text { Northern } \\
\text { Ireland] }\end{array}$ & Original research & $\begin{array}{l}\text { Medium- and } \\
\text { long-stay }\end{array}$ & $\begin{array}{l}\text { Guidelines (normative) } \\
\text { and Expert consensus }\end{array}$ & $\begin{array}{l}\text { Not to } \\
\text { reduce }\end{array}$ & Limited post-discharge support in the community \\
\hline Lesage and Tansella (81) & 1993 & Canada & $\mathrm{HI}$ & $\begin{array}{l}\text { Global } \\
\text { [Canada \& } \\
\text { Italy] }\end{array}$ & Original research & Long-stay & Guidelines (normative) & Reduce & $\begin{array}{l}\text { Reduced number of long-stay patients allows for further } \\
\text { bed removals }\end{array}$ \\
\hline Madianos (82) & 2002 & Greece & $\mathrm{HI}$ & National & Original research & Long-stay & Original estimate & Reduce & $\begin{array}{l}\text { Bed reductions, while maintaining personnel, improves } \\
\text { inpatient care conditions }\end{array}$ \\
\hline Okayama et al. (83) & 2020 & Japan & $\mathrm{HI}$ & National & Original research & Long-stay & Original forecast & Reduce & $\begin{array}{l}\text { Reduced number of long-stay patients allows for further } \\
\text { bed removals, follow global trends of psychiatric bed } \\
\text { reductions in most of the developed countries }\end{array}$ \\
\hline Sisti et al. (84) & 2015 & US & $\mathrm{HI}$ & National & Viewpoint & Long-stay & Expert opinion & Increase & $\begin{array}{l}\text { Need for the development of safe, modern and humane } \\
\text { asylums that provide long-term residential care for } \\
\text { people with severe mental illnesses }\end{array}$ \\
\hline \multicolumn{10}{|c|}{ Non-specified length of stay } \\
\hline Allison et al. (85) & 2017 & Australia & $\mathrm{HI}$ & $\begin{array}{l}\text { Global } \\
\text { [Australia, UK } \\
\text { and Canada] }\end{array}$ & Correspondence & Inpatient & Expert opinion & Increase & Increased detentions and bed pressure \\
\hline Bowersox et al. (86) & 2013 & US & $\mathrm{HI}$ & $\begin{array}{l}\text { National } \\
\text { VHA] }\end{array}$ & Original research & Inpatient & Original estimate & $\begin{array}{l}\text { Do not } \\
\text { change }\end{array}$ & $\begin{array}{l}\text { Short LOS and premature discharge, need for the } \\
\text { development of safe, modern and humane asylums that } \\
\text { provide long-term residential care for people with severe } \\
\text { mental illnesses }\end{array}$ \\
\hline Dazzan and Barbui (87) & 2015 & UK & $\mathrm{HI}$ & National & Editorial & Inpatient & Expert's opinion & $\begin{array}{l}\text { Not to } \\
\text { increase }\end{array}$ & $\begin{array}{l}\text { High occupancy, increase in involuntary admissions due } \\
\text { to lack of timely voluntary admission at an earlier stage of } \\
\text { illness, limited post-discharge in the community }\end{array}$ \\
\hline De Vetten et al. (88) & 2019 & Moldova & LMI & National & Original research & Inpatient & $\begin{array}{l}\text { Guidelines (normative) } \\
\text { and Expert consensus }\end{array}$ & Reduce & $\begin{array}{l}\text { New care pathways and better integration of emergency } \\
\text { departments, inpatient and outpatient services allow for } \\
\text { further psychiatric bed removals, follow global trends of } \\
\text { psychiatric bed reductions in most of the developed } \\
\text { countries }\end{array}$ \\
\hline Fioritti et al. (89) & 1997 & Italy & $\mathrm{HI}$ & $\begin{array}{l}\text { Local [Emilia- } \\
\text { Romagna] }\end{array}$ & Original research & Inpatient & Original estimate & Reduce & $\begin{array}{l}\text { New care pathways and better integration of emergency } \\
\text { departments, inpatient and outpatient services allow for } \\
\text { further psychiatric bed removals }\end{array}$ \\
\hline
\end{tabular}


TABLE 1 | Continued

\begin{tabular}{|c|c|c|c|c|c|c|c|c|c|}
\hline References & $\begin{array}{l}\text { Year of } \\
\text { publication }\end{array}$ & Country & $\begin{array}{l}\text { Income } \\
\text { group }\end{array}$ & $\begin{array}{l}\text { International, } \\
\text { national or } \\
\text { local scope } \\
\text { [catchment } \\
\text { area or } \\
\text { jurisdiction } \\
\text { when local] }\end{array}$ & $\begin{array}{l}\text { Type of } \\
\text { publication }\end{array}$ & $\begin{array}{l}\text { Length of } \\
\text { stay }\end{array}$ & $\begin{array}{l}\text { Approach to support } \\
\text { recommendation }\end{array}$ & $\begin{array}{l}\text { Suggested } \\
\text { trend of } \\
\text { psychiatric } \\
\text { bed } \\
\text { numbers }\end{array}$ & Argument for change per theme \\
\hline Fisher et al. (90) & 1996 & US & $\mathrm{HI}$ & $\begin{array}{l}\text { Local } \\
\text { [Massachusetts }\end{array}$ & $\begin{array}{l}\text { Original research } \\
\text { s] }\end{array}$ & Inpatient & Original estimate & Reduce & $\begin{array}{l}\text { Hospital bed numbers should be reduced to serve the } \\
\text { most severely ill patients }\end{array}$ \\
\hline Forchuk et al. (91) & 2006 & Canada & $\mathrm{HI}$ & $\begin{array}{l}\text { Local [London, } \\
\text { Ontario] }\end{array}$ & Original research & Inpatient & Original estimate & $\begin{array}{l}\text { Not to } \\
\text { reduce }\end{array}$ & $\begin{array}{l}\text { Short length of stay and premature discharge, discharge } \\
\text { to homelessness and shelters }\end{array}$ \\
\hline Forrester et al. (92) & 2013 & UK & $\mathrm{HI}$ & Local [London] & Original research & $\begin{array}{l}\text { Inpatient } \\
\text { (forensic) }\end{array}$ & Original estimate & Increase & $\begin{array}{l}\text { Delays in transferring individuals with mental disorders in } \\
\text { the criminal justice system to hospitals due to inpatient } \\
\text { bed shortage }\end{array}$ \\
\hline Geller and Biebel (93) & 2006 & US & $\mathrm{HI}$ & National & Original research & $\begin{array}{l}\text { Inpatient } \\
\text { (pediatric) }\end{array}$ & Original estimate & $\begin{array}{l}\text { Not to } \\
\text { reduce }\end{array}$ & $\begin{array}{l}\text { Lack of specialized psychiatric beds for children and } \\
\text { adolescents, increasing suicide rates, criminalization of } \\
\text { mentally ill }\end{array}$ \\
\hline Geng et al. (94) & 2020 & China & UMl & National & Original research & $\begin{array}{l}\text { Inpatient } \\
\text { (pediatric) }\end{array}$ & Original estimate & Increase & $\begin{array}{l}\text { Lack of specialized psychiatric beds for children and } \\
\text { adolescents }\end{array}$ \\
\hline Goldman and Keller (95) & 1978 & US & $\mathrm{HI}$ & National & Original research & Inpatient & Original estimate & Reduce & Low inpatient occupancy rates \\
\hline Guaiana et al. (10) & 2019 & $\begin{array}{l}\text { Australia \& } \\
\text { Canada }\end{array}$ & $\mathrm{HI}$ & Global & Correspondence & Inpatient & Expert opinion & $\begin{array}{l}\text { Do not } \\
\text { reduce }\end{array}$ & $\begin{array}{l}\text { High occupancy rates and overcrowding, overcrowding } \\
\text { and long waiting times in emergency departments, early } \\
\text { readmission rates, discharge to homelessness and } \\
\text { shelters, criminalization of mentally ill }\end{array}$ \\
\hline Hartvig and Kjelsberg (96) & 2009 & Norway & $\mathrm{HI}$ & National & Original research & Inpatient & Original estimate & Increase & Criminalization of mentally ill \\
\hline Hollander et al. (97) & 1996 & UK & $\mathrm{HI}$ & $\begin{array}{l}\text { Local [Greater } \\
\text { London, } \\
\text { England] }\end{array}$ & Letter & Inpatient & Expert opinion & Increase & $\begin{array}{l}\text { High occupancy rates and overcrowding, short length of } \\
\text { stay and premature discharge, hardships for patients } \\
\text { and families, compromised safety and occurrence of } \\
\text { serious incidents }\end{array}$ \\
\hline Hume and Rudin (98) & 1960 & US & $\mathrm{HI}$ & $\begin{array}{l}\text { Local } \\
\text { [California] }\end{array}$ & Original research & Inpatient & Guidelines (normative) & Increase & $\begin{array}{l}\text { Lack of specialized psychiatric beds for children and } \\
\text { adolescents }\end{array}$ \\
\hline Jeppesen et al. (99) & 2016 & Denmark & $\mathrm{HI}$ & National & Original research & $\begin{array}{l}\text { Inpatient } \\
\text { (schizophrenia) }\end{array}$ & Original estimate & Increase & $\begin{array}{l}\text { Increasing admission rates and waiting times, lack of } \\
\text { available inpatient beds and treatment for schizophrenia } \\
\text { patients }\end{array}$ \\
\hline Johnson (100) & 2011 & UK & $\mathrm{HI}$ & $\begin{array}{l}\text { National } \\
\text { [England \& } \\
\text { Wales] }\end{array}$ & $\begin{array}{l}\text { Short report } \\
\text { (opinion) }\end{array}$ & Inpatient & Expert opinion & $\begin{array}{l}\text { Do not } \\
\text { increase }\end{array}$ & Bed reductions reduce reliance on inpatient services \\
\hline Kaltiala-Heino et al. (101) & 2001 & Finland & $\mathrm{HI}$ & $\begin{array}{l}\text { Local (northern } \\
\text { Finland) }\end{array}$ & Original research & Inpatient & Original estimate & Increase & Short length of stay \\
\hline Keown et al. (102) & 2019 & UK & $\mathrm{HI}$ & $\begin{array}{l}\text { National } \\
\text { [England] }\end{array}$ & Original research & $\begin{array}{l}\text { Inpatient } \\
\text { (forensic) }\end{array}$ & Original estimate & Increase & Criminalization of mentally ill \\
\hline
\end{tabular}




\begin{tabular}{|c|c|c|c|c|c|c|c|c|c|}
\hline References & $\begin{array}{l}\text { Year of } \\
\text { publication }\end{array}$ & Country & $\begin{array}{l}\text { Income } \\
\text { group }\end{array}$ & $\begin{array}{l}\text { International, } \\
\text { national or } \\
\text { local scope } \\
\text { [catchment } \\
\text { area or } \\
\text { jurisdiction } \\
\text { when local] }\end{array}$ & $\begin{array}{l}\text { Type of } \\
\text { publication }\end{array}$ & $\begin{array}{l}\text { Length of } \\
\text { stay }\end{array}$ & $\begin{array}{l}\text { Approach to support } \\
\text { recommendation }\end{array}$ & $\begin{array}{l}\text { Suggested } \\
\text { trend of } \\
\text { psychiatric } \\
\text { bed } \\
\text { numbers }\end{array}$ & Argument for change per theme \\
\hline Kigozi et al. (103) & 2010 & Uganda & $\mathrm{LI}$ & National & Original research & Inpatient & Guidelines (normative) & Increase & High occupancy rates and overcrowding \\
\hline Kilsztajn et al. (104) & 2008 & Brazil & UMI & National & Original research & Inpatient & Original estimate & Decrease & $\begin{array}{l}\text { Reduce resources for inpatient care to develop } \\
\text { outpatient care }\end{array}$ \\
\hline Lamb (105) & 2015 & Latin America & HI \& LMI & South America & Editorial & Inpatient & Expert opinion & Increase & Criminalization of mentally ill \\
\hline Lawrence et al. (106) & 1991 & England & $\mathrm{HI}$ & $\begin{array}{l}\text { Local } \\
\text { [Kidderminster } \\
\text { District] }\end{array}$ & Original research & Inpatient & Guidelines (normative) & Maintain & $\begin{array}{l}\text { Sub-groups of people with severe mental illnesses are } \\
\text { still in need of psychiatric inpatient beds, implementation } \\
\text { of community care complements, but does not replace } \\
\text { inpatient care }\end{array}$ \\
\hline Lelliott and Audini (107) & 2003 & UK & $\mathrm{HI}$ & $\begin{array}{l}\text { Local [seven } \\
\text { local authority } \\
\text { areas, } \\
\text { England] }\end{array}$ & Original research & $\begin{array}{l}\text { Inpatient } \\
\text { (forensic, } \\
\text { young men) }\end{array}$ & Original estimate & Increase & $\begin{array}{l}\text { Early readmission rates, increasing detention rates due } \\
\text { to lack of adequate and timely mental health treatments } \\
\text { of persons with severe mental illnesses (and comorbid } \\
\text { substance use disorders) }\end{array}$ \\
\hline MacDonald (108) & 1989 & Italy & $\mathrm{HI}$ & Local (Rome) & Original research & Inpatient & Expert consensus & $\begin{array}{l}\text { Do not } \\
\text { reduce }\end{array}$ & Insufficient and ineffective community services \\
\hline Mundt et al. (8) & 2015 & $\begin{array}{l}\text { South } \\
\text { America }\end{array}$ & LMI & Global & Original research & Inpatient & Original estimate & Increase & Criminalization of mentally ill \\
\hline $\begin{array}{l}\text { Munk-Jorgensen and } \\
\text { Mortensen (109) }\end{array}$ & 1993 & Denmark & $\mathrm{HI}$ & National & Short report & $\begin{array}{l}\text { Inpatient } \\
\text { (schizophrenia) }\end{array}$ & Expert opinion & $\begin{array}{l}\text { Decrease is } \\
\text { possible } \\
\text { without } \\
\text { negative } \\
\text { effects }\end{array}$ & Decrease in first-ever admission rates of schizophrenia \\
\hline $\begin{array}{l}\text { Nilsson and Lögdberg } \\
\text { (110) }\end{array}$ & 2008 & Sweden & $\mathrm{HI}$ & Local [Malmö] & Original research & $\begin{array}{l}\text { Inpatient } \\
\text { (schizophrenia) }\end{array}$ & Original estimate & Increase & $\begin{array}{l}\text { Lack of available inpatient beds and treatment for } \\
\text { schizophrenia patients }\end{array}$ \\
\hline Nome and Holsten (111) & 2011 & Norway & $\mathrm{HI}$ & $\begin{array}{l}\text { Local } \\
\text { [Hordaland } \\
\text { County] }\end{array}$ & Original research & Inpatient & Original estimate & $\begin{array}{l}\text { Do not } \\
\text { reduce }\end{array}$ & $\begin{array}{l}\text { Implementation of community care complements, but } \\
\text { does not replace inpatient care }\end{array}$ \\
\hline Nordentoft et al. (112) & 1996 & Denmark & $\mathrm{HI}$ & $\begin{array}{l}\text { Local } \\
\text { [Copenhagen] }\end{array}$ & Original research & Inpatient & Original estimate & Increase & $\begin{array}{l}\text { Implementation of community care complements, but } \\
\text { does not replace inpatient care }\end{array}$ \\
\hline O'Neil et al. (113) & 2002 & Ireland & $\mathrm{HI}$ & National & Original research & $\begin{array}{l}\text { Inpatient } \\
\text { (forensic) }\end{array}$ & Original estimate & Increase & Criminalization of mentally ill \\
\hline
\end{tabular}




\begin{tabular}{|c|c|c|c|c|c|c|c|c|c|}
\hline References & $\begin{array}{l}\text { Year of } \\
\text { publication }\end{array}$ & Country & $\begin{array}{l}\text { Income } \\
\text { group }\end{array}$ & $\begin{array}{l}\text { International, } \\
\text { national or } \\
\text { local scope } \\
\text { [catchment } \\
\text { area or } \\
\text { jurisdiction } \\
\text { when local] }\end{array}$ & $\begin{array}{l}\text { Type of } \\
\text { publication }\end{array}$ & $\begin{array}{l}\text { Length of } \\
\text { stay }\end{array}$ & $\begin{array}{l}\text { Approach to support } \\
\text { recommendation }\end{array}$ & $\begin{array}{l}\text { Suggested } \\
\text { trend of } \\
\text { psychiatric } \\
\text { bed } \\
\text { numbers }\end{array}$ & Argument for change per theme \\
\hline Ose et al. (9) & 2018 & Norway & $\mathrm{HI}$ & National & Original research & Inpatient & Original estimate & $\begin{array}{l}\text { Do not } \\
\text { reduce }\end{array}$ & Insufficient and ineffective community services \\
\hline Roberts et al. (114) & 2014 & Ghana & LMI & National & Original research & Inpatient & Original estimate & Increase & High occupancy rates and overcrowding \\
\hline Rothbard et al. (115) & 1998 & US & $\mathrm{HI}$ & $\begin{array}{l}\text { Local } \\
\text { [Philadelphia] }\end{array}$ & Original research & Inpatient & Original estimate & Increase & $\begin{array}{l}\text { Higher total health care system costs due to lack of beds } \\
\text { (queuing in General Hospitals) }\end{array}$ \\
\hline Sasaki (116) & 2012 & Japan & $\mathrm{HI}$ & Local & Original research & Inpatient & Expert opinion & Reduce & $\begin{array}{l}\text { Economic incentives for inadequately long inpatient bed } \\
\text { use }\end{array}$ \\
\hline Someya et al. (117) & 2004 & Japan & $\mathrm{HI}$ & $\begin{array}{l}\text { Local [Niigata } \\
\text { Prefecture] }\end{array}$ & Original research & $\begin{array}{l}\text { Inpatient } \\
\text { (schizophrenia) }\end{array}$ & Original forecast & Reduce & $\begin{array}{l}\text { Trend analyses show less psychiatric bed needs of } \\
\text { schizophrenia patients }\end{array}$ \\
\hline Svab et al. (118) & 2006 & Slovenia & $\mathrm{HI}$ & National & Short report & Inpatient & Expert opinion & Increase & Long waiting lists for outpatient services \\
\hline $\operatorname{Tim}(75)$ & 2013 & UK & $\mathrm{HI}$ & National & Editorial & $\begin{array}{l}\text { Inpatient } \\
\text { (forensic) }\end{array}$ & Expert opinion & Increase & $\begin{array}{l}\text { Increasing detention rates due to lack of adequate and } \\
\text { timely mental health treatments of persons with severe } \\
\text { mental illnesses (and comorbid substance use disorders) }\end{array}$ \\
\hline Torrey et al. (119) & 2012 & US & $\mathrm{HI}$ & National & $\begin{array}{l}\text { Report (Treatment } \\
\text { Advocacy Center) }\end{array}$ & Inpatient & Expert opinion & $\begin{array}{l}\text { Do not } \\
\text { reduce }\end{array}$ & $\begin{array}{l}\text { Overcrowding and long waiting times in emergency } \\
\text { departments, sub-groups of people with severe mental } \\
\text { illnesses are still in need of psychiatric inpatient beds, } \\
\text { criminalization of mentally ill }\end{array}$ \\
\hline Trieman and Leff (120) & 1996 & UK & $\mathrm{HI}$ & $\begin{array}{l}\text { Local [North } \\
\text { London] }\end{array}$ & Original research & Inpatient & Original estimate & Reduce & Inpatient services are restrictive environments \\
\hline Weller and Weller (121) & 1988 & England & $\mathrm{HI}$ & Local [London] & Original research & $\begin{array}{l}\text { Inpatient } \\
\text { (forensic) }\end{array}$ & Expert opinion & Increase & Criminalization of mentally ill \\
\hline $\begin{array}{l}\text { Worrall and O'Herlihy } \\
\text { (122) }\end{array}$ & 2001 & UK & $\mathrm{HI}$ & National & Original research & $\begin{array}{l}\text { Inpatient } \\
\text { (pediatric) }\end{array}$ & Expert consensus & Increase & $\begin{array}{l}\text { Overcrowding and long waiting times in emergency } \\
\text { departments, lack of specialized psychiatric beds for } \\
\text { children and adolescents }\end{array}$ \\
\hline Yoon and Bruckner (123) & 2009 & US & $\mathrm{HI}$ & National & Original research & Inpatient & Original estimate & $\begin{array}{l}\text { Do not } \\
\text { reduce }\end{array}$ & $\begin{array}{l}\text { Increasing suicide rates, insufficient and ineffective } \\
\text { community services }\end{array}$ \\
\hline Yoon et al. (124) & 2013 & US & $\mathrm{HI}$ & $\begin{array}{l}\text { Local [King } \\
\text { County, } \\
\text { Washington] }\end{array}$ & Original research & $\begin{array}{l}\text { Inpatient } \\
\text { (forensic, SMI) }\end{array}$ & Original estimate & $\begin{array}{l}\text { Do not } \\
\text { reduce }\end{array}$ & $\begin{array}{l}\text { Increasing detention rates due to lack of adequate and } \\
\text { timely mental health treatments of persons with severe } \\
\text { mental illnesses (and comorbid substance use disorders) }\end{array}$ \\
\hline
\end{tabular}

ED, emergency department; GHPU, general hospital psychiatric units; LOS, length of stay; HI, High-Income; SMI, severely mentally ill patients; UK, United Kingdom; USA, United States of America; UMI, Upper Middle-Income; LMI, Low- and Medium-Income; VHA, veterans health administration. 
TABLE 2 | Number of expert arguments per theme and country.

\begin{tabular}{ll} 
Themes $\quad$ World Bank Income classification \\
\hline
\end{tabular}

High-Income Countries (HIC)

Low- and Middle-Income Countries (LMIC)

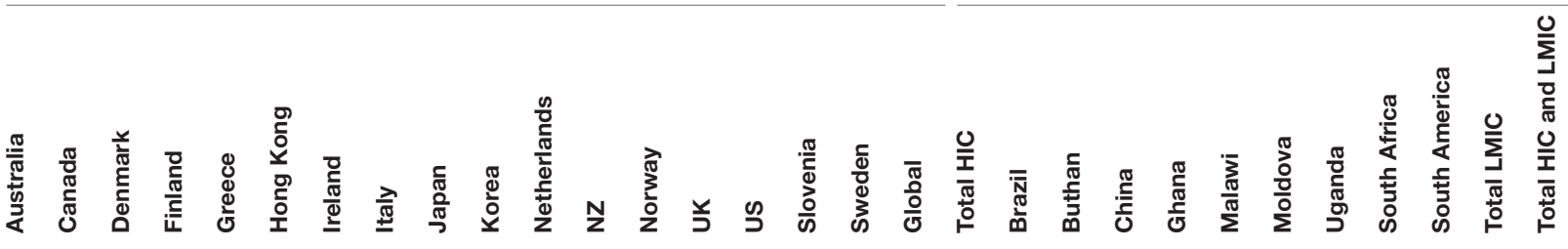

\section{Expert arguments to reduce psychiatric bed numbers}

1.1. Cost effectiveness

1.1.1. Lower overall cost of home-based treatment compared with inpatient services

1.1.2. Implementation of a day hospital service and home

treatment teams allows for greater concentration of inpatien

resources on most severely ill patients, leading to cost savings

1.1.3. Reduce resources for inpatient care to develop

outpatient care

1.2. Inappropriate use of inpatient care

1.2.1. Inappropriately long psychiatric inpatient care

1.2.2. Reduced number of long-stay patients allows for

further psychiatric bed removals

1.2.3. Inpatient psychiatric bed capacity and availability

generates utilization and coercive treatments

1.2.4. Economic incentives for inadequately long inpatient

1.3. Bed reductions lead to better use and development of existing community care

1.4. Quality of care is maintained or improved with less beds

1.4.1. Bed reductions, while maintaining personnel, improves inpatient care conditions

1.4.2. Bed reductions do not affect the quality of care in the

system as a whole and has not shown negative effects

1.5. Less psychiatric bed needs

1.5.1. Trend analyses show less psychiatric bed needs of

schizophrenia patients

1.5.2. Decrease in first-ever admission rates of schizophrenia

1.5.3. Low inpatient occupancy rates

1.6. Inpatient services are restrictive environments

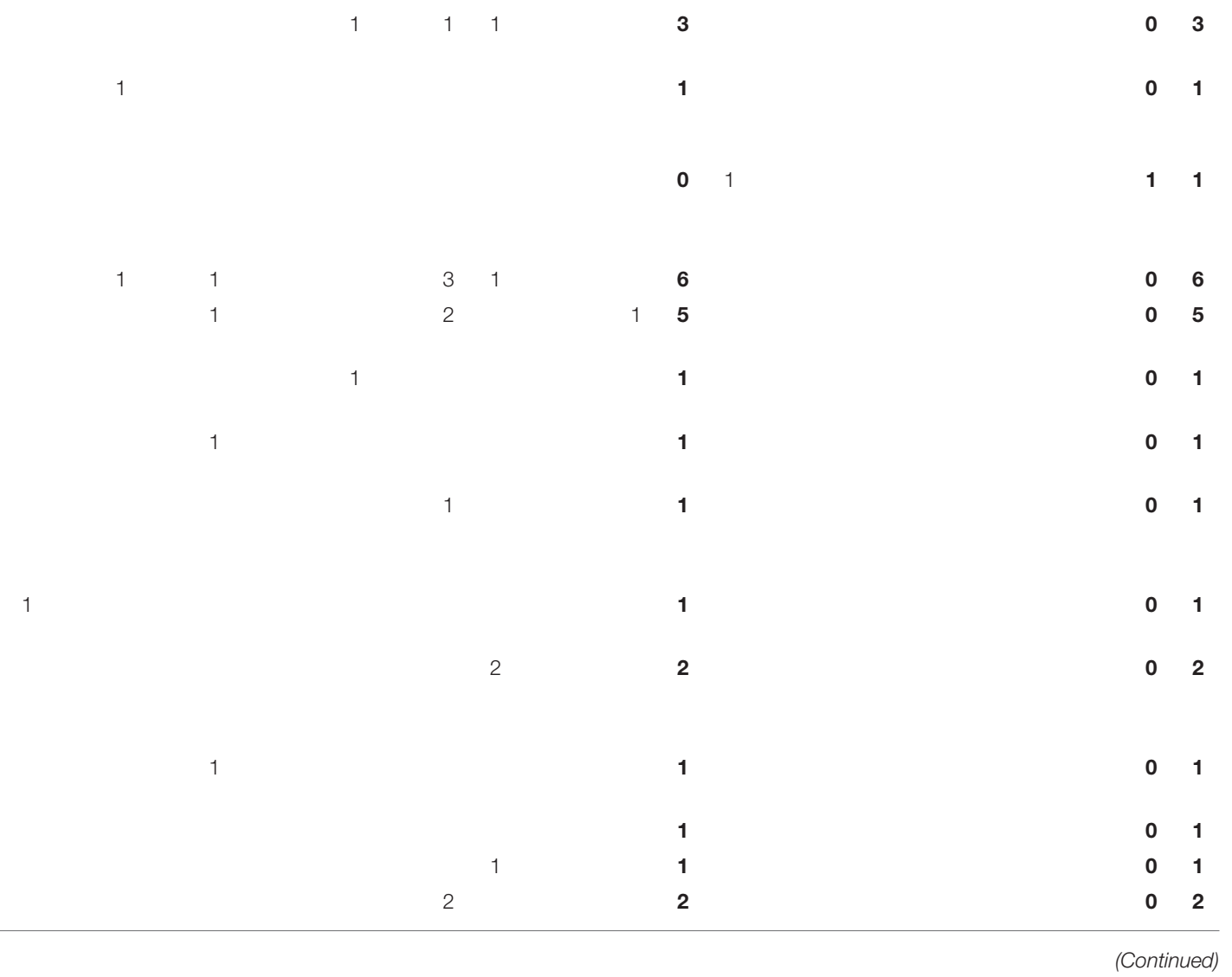


1.7. New care pathways and better integration of emergency 1 departments, inpatient and outpatient services allow for further psychiatric bed removals

1.8. Follow global trends of psychiatric bed reductions in most of the developed countries

1.9. Bed reductions reduce reliance on inpatient services

1.10. Hospital bed numbers should be reduced to serve the most severely ill patients

Total

\section{Expert arguments to increase or maintain psychiatric bed numbers}

2.1. Lack of beds for financial pressure

2.1.1. Financial pressure on the mental health system has resulted in too many bed removals and underfunded inpatient care systems

2.1.2. Financial disincentives and unfair reimbursement

practice have led to lower numbers of psychiatric beds than actually needed

2.2. Higher total health care system costs due to bed

closures (queuing in General Hospitals)

2.3. High demand of psychiatric beds

2.3.1. High occupancy rates and overcrowding

2.3.2. Increasing admission rates and waiting times

2.3.3. Overcrowding and long waiting times in emergency

departments

2.4. Inadequately short length of stay

2.4.1. Short length of stay and premature discharge

2.4.2. Revolving door effect: Early readmission rates

2.5. Lack of specialized psychiatric beds for children and

adolescents

2.6. Lack of locally available beds

2.6.1. Need for the development of integrated health care

systems with decentralized inpatient care capacities

numbers

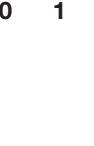






2.6.2. Risk of transfer outside patients' local community for

care

2.7. Lack of beds compromises quality of care

2.7.1. Hardships for patients and families, compromised

safety and occurrence of serious incidents

2.7.2. Severe emotional and physical harm to patients, 1

families and communities

2.8. Increase in involuntary admissions due to lack of timely

voluntary admission at an earlier stage of illness

2.9. Increasing suicide rates

2.10. Sub-groups of people with severe mental illnesses are

still in need of psychiatric inpatient beds

2.10.1. Need for the development of safe, modern and

people with severe mental illnesses

2.10.2. Lack of available inpatient beds and treatment for

schizophrenia patients

2.11. Insufficient and ineffective community services

2.11.1. Limited post-discharge support in the community

2.11.2. Long waiting lists for outpatient services

2.11.3. Implementation of community care complements, but does not replace inpatient care

2.12. Lack of affordable and supported housing services

2.12.1. Discharge to homelessness and shelters

2.13. Criminalization of mentally ill

2.13.1. Increasing detention rates due to lack of adequate

and timely mental health treatments of persons with severe

2.13.2. Delays in transferring individuals with mental disorders shortage

Total

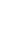

humane asylums that provide long-term residential care for mental illnesses (and comorbid substance use disorders)

in the criminal justice system to hospitals due to inpatient bed

(1)

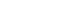

NZ, New Zealand; UK, United Kingdom; US, United States of America; HIC, High- and upper-middle income countries; LMIC, Low- and Middle-Income countries. 
"The combination of adding a home treatment team and halving the number of inpatient beds was, when compared to a control area, associated with (a) additional numbers of people receiving acute care (b) a lower cost per individual and (c) no difference in overall service cost" (37)

Implementation of day hospital services and home "The major reduction in the number of acute inpatient beds and the opening of an acute day hospital resulted treatment teams allow for greater concentration of in greater concentration of inpatient resources on the more severely ill patients" (64) inpatient resources on most severely ill patients, leading to cost savings

Reduce resources for inpatient care to develop outpatient care

"The precarious extra-hospital network has been used as a barrier to deactivation of psychiatric beds, although the latter generates the necessary resources for the former" (104)

2. Inappropriate use of inpatient care

Inappropriately long psychiatric inpatient care

Reduced number of long-stay patients allows for further bed removals

Inpatient psychiatric bed capacity and availability generates utilization and coercive treatments

"36\% of patients do not need to be in hospital if appropriate after-care could be found" (33)

"Alternatives to the mental hospital exist and may limit the use of long stay hospital beds through comprehensive community care that also includes proper residential provisions" (81)

"Substantially lower rates of bed provision than those currently provided, with the concomitant development of a wide range of community based services could do much to prevent the current excessive tendency to commit patients and to the fostering of disability and dependency which perpetuates the continuing need for such beds" (57)

Economic incentives for inadequately long inpatient "It will be necessary in the future to transit from a medical fee system that promotes long-term hospitalization bed use and large-scale expansion to one in which downsizing correlates with better financial results" (116)

3. Bed reductions lead to better use of existing

community care

4. Quality of care is maintained or improved with Bed reductions, while maintaining personnel, less beds improves inpatient care conditions

"Discharge of new long stay patients within a psychiatric service that is community-oriented, support patients in their own homes and make the fullest possible use of non-hospital residential and nursing homes" (79)

"The reduction in the number of beds in the public psychiatric sector has led to significant improvement in nursing conditions" (82)

Bed reductions do not affect the quality of care in "A $50 \%$ reduction in acute beds - and a 23\% reduction in total beds - on an inpatient service that had been the system as a whole and have not shown negative operating at full capacity was not associated with anticipated negative effects, such as increased demand for effects psychiatric emergency services, decreased access to emergency or inpatient services, increased recidivism to inpatient care, or increased levels of inadequately treated mental illness in the community" (6)

5. Psychiatric bed needs have been overestimated

Trend analyses show less psychiatric bed needs of "Present results showing a reduction of the number of schizophrenic inpatients to two-fifths of the present schizophrenia patients

Decrease in first-ever admission rates of

schizophrenia number is significant for hospital planning and healthcare resource allocation" (117)

"First-ever admission rates of schizophrenia in Denmark have decreased since 1970. The most obvious explanation is the extensive restructuring of the psychiatric service of which a decrease in available beds of more than $50 \%$ seems to be most important" (109)

Low inpatient occupancy rates

"The most apparent consequence of this ineffective planning is that many centers have more beds than they require. A third of the centers in our sample had occupancy rates of 50\% or less" (95)

"We conclude that greater emphasis and urgency needs to be placed on moving patients on from acute mental health units after 9 weeks of admission. This can lead to more appropriate care for patients in less restrictive environments and reduce demand on acute psychiatric units and reduce the necessity and stress to patients and careers of acute admissions far from home" (48) 
7. New care pathways and

better integration of

emergency departments,

inpatient and outpatient

services allow for further

psychiatric bed removals

8. Countries should follow

global trends to reduce

psychiatric beds

9. Bed reductions reduce

reliance on inpatient

services

\section{Hospital bed numbers}

can be reduced to serve the

most severely ill patients

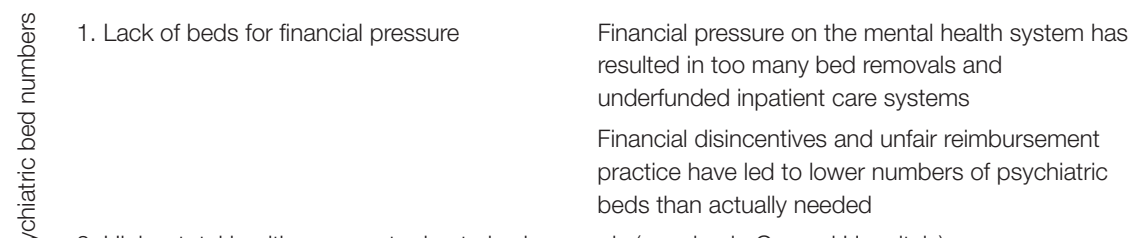

2. Higher total health care costs due to bed removals (queuing in General Hospitals)

3. High demand of psychiatric beds

Increasing admission rates and waiting times

Overcrowding and long waiting times in emergency

4. Inadequately short length of stay
High occupancy rates and overcrowding

departments beds and community services have decreased" (27)

"Our study demonstrated that reducing beds and introducing new care pathway interventions in inpatient and community settings are associated with better ward practices and improvements in patient flow between the emergency department, the inpatient ward and community teams" (5)

"In Korea, however, admission remains the foremost resource in psychiatric treatment. In contrast with the general trend in most developed countries, the number of psychiatric beds in Korea has continuously increased, and the length of stay of psychiatric patients in Korea has remained long for years" (80)

"Thus increasing psychiatric bed provision would, in the current climate of scarcity, be both profligate and pointless. Let us instead dedicate the limited resources we have to improving the quality of existing inpatient services and increasing their acceptability to patients, and to implementing as fully as we can the knowledge that we already have about how reliance on inpatient services may be reduced" (100)

"Our data suggest that an ever broader spectrum of persons with severe mental illness can be managed in the community as more community-based and alternative inpatient settings are created to meet their needs. But the most difficult populations remain, and they appear resistant to permanent exclusion from the state hospital, even in the best-funded community systems" (90)

"There is a risk that the significant financial pressures on mental health trusts can result in too many bed closures" (45)

"Specific emphasis should be placed on lobbying for fair reimbursement of services, including psychiatric emergency and inpatient services, as care places a financial strain on hospitals, thus providing a disincentive for hospitals to keep units open or add to existing services" (63)

"Despite the decreased number of extended care days and the increased supply of residential care slots, individuals having acute care episodes that required hospitalization had higher episode and annual costs in the post (state hospital) closure period. The data suggest that the increased costs were due primarily to the increased use of acute care general hospital days that were the consequence of patients queuing up in general hospitals while waiting for a transfer to an intermediate care unit" (115)

"In addition, many in-patient wards now regularly have a 100-120\% occupancy rate, which is significantly higher than the $85 \%$ recommended by the Royal College of Psychiatrists" (87)

"Findings of the study indicate that psychiatric admissions in psychiatric ward are increasing year after year" (68)

Short length of stay and premature discharge "The average length of stay, varying from less than a week in the USA to 15 days in the UK, is inadequate for adequate assessment or treatment. ...hospital managers spend a large proportion of their time juggling the relative risks of discharging patients prematurely or delaying admission" (73) 
Revolving door effect: Early readmission rates

5. Lack of specialized psychiatric beds for children and adolescents

6. Lack of locally available beds

7. Lack of beds compromises quality of care for care safety and occurrence of serious incidents families and communities

8. Increase in involuntary admissions due to lack of timely voluntary admission at an earlier stage of illness

9. Psychiatric beds may prevent suicide in people with psychosis

10. Sub-groups of people with severe mental

ilnesses are still in need of psychiatric inpatient beds humane asylums that provide long-term residentia care for people with severe mental illnesses schizophrenia patients
"Length of stay and the crisis discharge policy seem to exacerbate the revolving door effect in this psychiatric hospital. Readmission is often used as quality indicator for inpatient psychiatric services, and could be seen as a failure of the earlier hospital admission" (62)

"In Wales no psychiatrist has access to an adolescent psychiatric in-patient bed for emergency admissions" (31)

Need for the development of integrated health care "In broad terms, the study recommends an increase in the number of acute psychiatric beds in genera systems with decentralized inpatient care capacities hospitals; development of community-based residential care; redistribution of staff from hospital to community services, particularly in rural areas; and the development of information systems to monitor the transitions to community-based care" (55)

Risk of transfer outside patients' local community "Inadequate local and regional psychiatric hospital (bed) capacity results in significantly prolonged emergency department length of stay and puts many patients at risk for transfer outside their local community for care" (66)

Hardships for patients and families, compromised

Severe emotional and physical harm to patients,

"Demoralization of patients and staff, with premature discharges and patients being placed inappropriately in isolating bed and breakfast or hostel accommodation with untrained or ill prepared staff. Under such circumstances, conditions are ripe for the occurrence of serious incidents" (97)

"If a person in need is unable to access an acute bed, severe emotional or at times physical harm to them and their career or family is a potential or high risk and can affect the wider community" (125)

"The reduction in beds has been matched by a parallel, $>60 \%$ increase in involuntary admissions during the same period, which does not seem to be matched by an increase in national mental health disorders and is possibly related to increase symptom severity at the time of presentation" (87)

"There has been a 20\% increase in Australian suicide rates over the decade 2006-2016" (29)

"The lack of change in bed use supports the view that there is a 'bed-rock' of serious illness which will always need in-patient care" (106)

Need for the development of safe, modern and

"This was the original meaning of psychiatric "asylum" - a protected place where safety, sanctuary, and

Lack of available inpatient beds and treatment for long-term care for the mentally ill would be provided. It is time to build them-again" (84)

"This population-based investigation showed an increase over time in the number and proportion of patients with schizophrenia who were not discovered until many days after death, which was correlated with the decrease in the number of available hospital beds for this group of patients" (110) 
11. Insufficient and ineffective community services

\section{Lack of affordable and supported housing} services

13. Criminalization of mentally ill
"The monies saved in closing psychiatric institutions and moving (too few) beds into the general hospitals were to be redirected to effective community programmes, but this has largely not occurred" (29)

Limited post-discharge support in the community "This could indicate that hospitals are allocating scarce beds to the most vulnerable patients, or that it is more challenging to accelerate the discharges of older patients, for example due to limitations in the availability of post-discharge support in the community" (38)

Long waiting lists for outpatient services

"The waiting time for outpatient psychiatric treatment in the central Slovenian region has been increasing

presently being 4 months on average. The access to psychiatric outpatient facilities, which used to be easy in the past even without referral forms, is becoming now increasingly difficult" (118)

Implementation of community care complements, "Our brief review of the literature on community based residential alternatives to acute psychiatric care but does not replace inpatient care suggests that these services are not alternatives for all patients, and as such are not completely substitutable for acute care" (67)

"Discharge from psychiatric wards to shelters or the streets is a real problem "/" Practitioners need to recognize that a shelter is not an appropriate 'address' for discharging individuals recovering from mental illness." (91)

"(...) the shortage of public psychiatric beds contributes to a number of costly and sometimes dangerous social problems, including jails and prisons overcrowded with inmates who are acutely ill and untreated" (119)

Increasing detention rates due to lack of adequate "Funding more psychiatric beds would reduce the detention rates by allowing timely voluntary admission to a and timely mental health treatments of persons with local acute psychiatric bed at an earlier stage of illness" (85) severe mental illnesses (and comorbid substance use disorders)

Delays in transferring individuals with mental

"More secure psychiatric beds may be required, at least in the short term, to support diversion policies and disorders in the criminal justice system to hospitals enable compliance with national policy directive, and to establish whether redesigned pathways can enhance due to inpatient bed shortage 


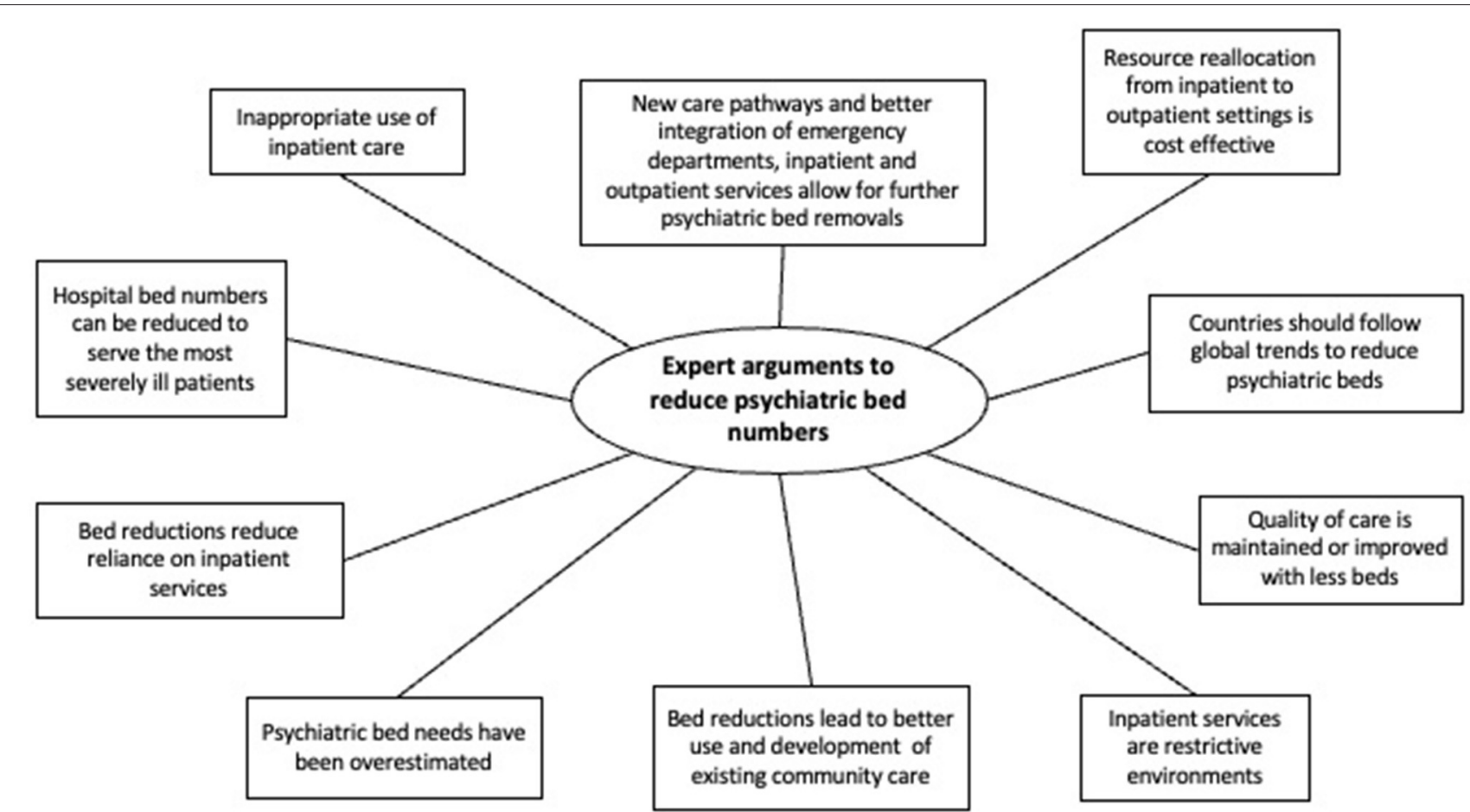

FIGURE 2 | Expert arguments to reduce psychiatric bed numbers, a systematic review of qualitative data.

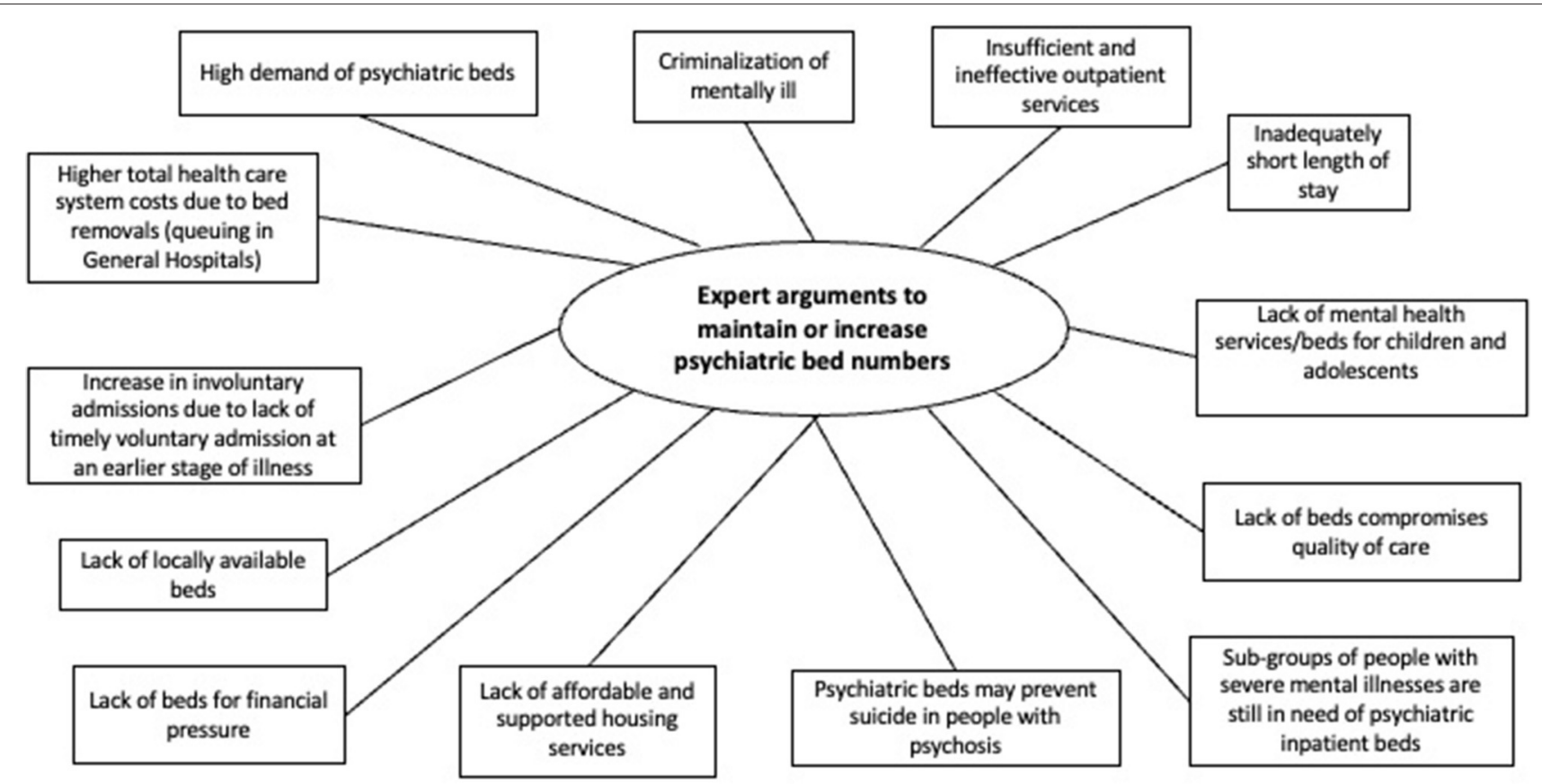

FIGURE 3 | Expert arguments to maintain or increase psychiatric bed numbers, a systematic review of qualitative data.

Inpatient Care Has Inappropriately Long Duration

" $36 \%$ of patients do not need to be in hospital if appropriate after-care could be found" (33), which often leads to an inappropriately long use of inpatient care. Reducing long-stay patients allows for further bed removals, especially considering that inpatient bed capacity and availability generates utilization 
and coercive treatments. However, there are economic incentives for inadequately long inpatient bed use $(39,40,48,57,64,71,78$, $79,81,83,116)$.

\section{New Care Pathways With Better Integration of Emergency Departments, Inpatient and Outpatient Services Allow for Further Psychiatric Bed Removals}

Developing an integrated and well-balanced health care system combining acute and community-based care has shown improved outcomes, such as better ward practices and improvements in patient flow between the different mental health services $(5,39,88)$.

\section{Resource Reallocation From Inpatient to Outpatient Settings Is Cost Effective}

Better financial and treatment outcomes can be achieved by reallocating resources within the mental health system, as the cost of home-based treatments is lower. Resources reallocated from inpatient services can be used to develop outpatient care. The implementation of day hospital services and home treatment teams allows for a greater concentration of inpatient resources on the most severely ill patients, leading to cost savings (40, 57, 64, 104). "The combination of adding a home treatment team and halving the number of inpatient beds was, when compared to a control area, associated with (a) additional numbers of people receiving acute care (b) a lower cost per individual and (c) no difference in overall service cost" (37).

\section{Countries Should Follow Global Trends to Reduce Psychiatric Beds}

In several countries with comparatively high numbers of psychiatric beds or high reliance on psychiatric beds within the service system as in Korea, Japan and Moldova, the call to follow global trends of psychiatric bed reductions has been made $(80,83,88)$.

\section{Quality of Care Is Maintained or Improved With Less Beds}

No anticipated negative effects have been associated with bed reductions. On the contrary, bed reductions, while maintaining personnel, improves inpatient care conditions. Statements included decongestion of wards, appropriate staffing and training and development of additional programs contributing to better treatment $(6,28,82)$.

\section{Inpatient Services Are Restrictive Environments}

The transfer of patients from acute to community care allows for treatment in a more adequate setting, which could improve aggressive behavior and reduces stress of patients and careers generated by acute admissions far from home $(48,120)$.

\section{Bed Reductions Lead to Better Use of Existing Community Care}

Reducing long-term hospitalization foments treatments outside of hospital facilities, possibly in their own homes (79).

\section{Psychiatric Bed Needs Have Been Overestimated}

Ineffective planning of beds and lack of understanding of acute care has led to an overestimation for the number of beds needed.
Statements included low occupancy rates, and trend analyses showing less psychiatric bed need and a decrease in first-ever admission rates of schizophrenia patients $(95,109,117)$.

\section{Bed Reductions Reduce Reliance on Inpatient Services}

Increasing psychiatric bed numbers would be pointless as bed reductions reduce dependence on inpatient care. There is a need to focus scarce resources on improving the quality of care of existing inpatient services (100).

\section{Hospital Bed Numbers Should Be Reduced to Serve the Most Severely Ill Patients}

Inpatient care should focus on acute care for the most severely ill patients. However, inpatient psychiatric beds should not be eliminated as this subgroup cannot be successfully treated in the community (90).

\section{Expert Arguments to Maintain or Increase Psychiatric Bed Numbers}

Regarding the second category of expert arguments to maintain or increase psychiatric bed numbers, thirteen themes emerged.

\section{High Demand of Psychiatric Beds}

Deinstitutionalization has resulted in increased occupancy rates and overcrowding, frequently over $100 \%$, along with increasing admission rates and waiting times in both HICs and LMICs (10, $25,27,30,32,36,41,43,44,46,49,50,52,54,56,59,60,65,66$, $68,69,74,87,99,103,114,119,122,126)$. "Mental health services in Australia, Canada, the UK, and the USA are all struggling to meet the demand for bedded care and are experiencing negative outcomes such as out of catchment admissions, access blocks in emergency departments, excessively high ward occupancy, discharge to homelessness, criminalization of the mentally ill, and early readmission" (10).

\section{Criminalization of Mentally Ill}

Increasing detention rates result from a lack of adequate and timely mental health treatment of persons with severe mental illnesses (and comorbid substance use disorders), as well as delays in transferring individuals with mental disorders in the criminal justice system to hospitals due to bed shortage $(8,10,47,59$, $70,75,85,92,93,96,102,105,107,119,124)$. One publication reports: "Because of the lack of accessible and effective services for those with serious mental illnesses, patients are falling through the net of psychiatric services and are imprisoned for minor public order offenses" (113).

\section{Insufficient and Ineffective Community Services}

There has been a wide gap between the closure of inpatient beds and the development of alternative care, which has resulted in limited post-discharge support in the community and long waiting lists for outpatient services. One study mentions: "The monies saved in closing psychiatric institutions and moving (too few) beds into the general hospitals were to be redirected to effective community programmes, but this has largely not occurred" (29). In addition, the implementation of community care complements, but does not replace inpatient care. Even where decentralized services have been developed, it still appears 
that there are too few inpatient beds to adequately treat acutelyill patients $(9,14,34,35,38,42,50,52,53,59,67,72,73,87,106$, $108,111,118,123)$.

\section{Inadequately Short Length of Stay}

Premature discharges have led to early readmission rates, frequently referred to as the "revolving door effect", which often results in patients being inappropriately placed in extra-hospital accommodation with untrained staff $(10,25,38,62,72,73,86,91$, 97, 99, 101, 107).

\section{Lack of Specialized Psychiatric Beds for Children and Adolescents}

Mental health professionals have to "borrow" beds from adult services or considerably delay treatment, which in turn leads to increased emotional disorders, suicide rates, drug overdose and criminalization of young people with mental illness $(31,51,72$, 93, 94, 98, 122).

\section{Lack of Beds Compromises Quality of Care}

Significant hardships for patients and families compromises their safety and increases the probability of the occurrence of serious incidents, along with severe emotional and physical harm to them and their families $(26,27,51,65,66,73,125)$. One publication reports: "Demoralization of patients and staff, with premature discharges and patients being placed inappropriately in isolating bed and breakfast or hostel accommodation with untrained or ill prepared staff. Under such circumstances, conditions are ripe for the occurrence of serious incidents" (97).

\section{Sub-Groups of People With Severe Mental Illnesses Are Still in Need of Psychiatric Inpatient Beds}

This small group of patients have shown an inability to be treated in non-acute settings. In addition, there is a need to develop safe, modern and humane asylums that provide long-term residential care for the severely mentally ill $(47,70,77,84,86,99,110,119)$. One study reports: "The lack of change in bed use supports the view that there is a 'bed-rock' of serious illness which will always need inpatient care" (106).

\section{Psychiatric Beds May Prevent Suicide in People With Psychosis}

There has been an increase of suicide rates between 20 and 100\% in people with non-organic psychosis. Providing psychiatric beds during crisis may prevent suicide in this population $(29,59,93$, 123).

\section{Lack of Affordable and Supported Housing Services}

Delayed admissions and early discharges lead to patients being discharged to homelessness, as they cycle through emergency departments, shelters and criminal settings without receiving adequate treatment $(10,36,91,119)$.

\section{Lack of Beds for Financial Pressure}

Financial disincentives and unfair reimbursement practices have led to lower numbers of psychiatric beds than actually needed $(49,63)$. "There is a risk that the significant financial pressures on mental health trusts can result in too many bed closures" (45).

\section{Lack of Locally Available Beds}

There is a need to decentralize inpatient services from urban to rural areas, as this uneven distribution of beds has resulted in prolonged lengths of stay in emergency departments, along with a higher risk of transfer outside of patients' community for care leading to significant hardships as they have to travel long distances to access treatment $(55,66,73,76)$.

\section{Increase in Involuntary Admissions Due to Lack of Timely \\ Voluntary Admission at an Earlier Stage of Illness}

Delayed admissions and premature discharges end up in involuntary longer lengths of stay possibly due to increased symptom severity at intake $(73,87)$.

\section{Higher Total Health Care System Costs Due to Bed Removals (Queuing in General Hospitals)}

Patients in acute care who are waiting for transfers to an intermediate care unit are generating bed blocks in general hospitals, thus increasing total costs (115).

\section{DISCUSSION}

\section{Main Findings}

This systematic review on expert arguments regarding trends of psychiatric bed numbers was based on 106 publications from 25 countries, including 14 studies from LMICs. First, there was not any general agreement on the direction of trends that should be pursued in policies, as there were arguments for reductions as well as for increases of psychiatric bed rates. Secondly, a complex matrix of partially contradicting arguments emerged, i.e., with respect to costs and quality of care. In the absence of hard evidence, those arguments remain relevant and have to be carefully considered in specific contexts for local policies.

\section{Comparison With the Literature}

Mental health service planners need orientation on how to further develop the psychiatric inpatient sector (7-10). Despite the diversity of services and nomenclatures used across regions $(10,16-18)$, there was agreement on several themes, such as the need to develop integrated mental health systems that assure coherence and continuity of care, along with the need to aim for specific populations and contexts (127). In all, arguments expressing concern about further bed reductions prevailed as there is a high demand on inpatient services, especially on short-stay and acute beds, evidenced in high occupancy and increasing admission rates $(10,99)$. In the US and Australia, overcrowding and long waiting times in emergency departments showed to be particularly relevant $(25,27,30,43,52,60,66$, 119). The relevance of psychiatric beds for problems that arise outside of the health system, such as violent crime of people with mental illnesses, increased detention rates and discharge to homelessness due to a lack of timely treatment and admission, was considered in several HICs $(36,61,85,128)$. Criminalization of mentally ill consistently appeared as a relevant theme in the US $(10,70,93,119)$ and was also mentioned for Latin America $(8,105)$. Inadequately short lengths of stay emerged as an issue in HICs (73), and in South Africa among LMICs (62). 
Mental health professionals are forced to prematurely discharge patients in order to free already scarce beds risking short readmission intervals, referred to as the revolving door effect (99). Several authors have expressed their concern that there are gaps in the mental health system due to major reductions of inpatient bed capacities that have been implemented without the appropriate development of community care $(52,129)$. There was agreement for HICs and LMICs that insufficient provision of outpatient services may not compensate for more bed reductions, resulting in overcrowding of inpatient facilities, which subsequently compromises the quality care (99). Several authors therefore call to halt current trends to further remove beds. Within this context, limited post discharge support in the community showed to be an important point in the UK $(14,38$, 50,87 ), and the lack of specialized psychiatric beds for children and adolescents emerged as particularly relevant in the UK and the US. Other subspecialty beds, such as mother baby units, may also be lacking, especially in LMICs (130). This overall lack of beds compromises patient safety and quality of care, carrying hardships for patients and families, and may provoke serious incidents (97). Some arguments emerged for specific contexts, such as increasing suicide rates related to major bed reductions, especially in the US $(93,123)$, and the argument that financial pressures on the mental health system have resulted in too many bed removals in Greece and the UK $(45,49)$.

In regard to arguments supporting the further reduction of psychiatric beds, most authors referred to long-stay beds and to inappropriately long lengths of stay $(71,78,79,81$, 83), emphasizing that patients could be transferred to the community if more timely and adequate after-care could be found $(33,39,40,48,64)$. The paradigm emerged that successful development of effective community services, including housing, and a better integrated mental health system would allow to continue psychiatric bed removals without negative outcomes (4-6), especially considering that inpatient services can be restrictive environments (48). We observed that a further reduction of psychiatric beds was usually not recommended for LMICs, except for Moldova (88). Several authors identified a need to follow trends of psychiatric bed reductions, which were enforced in most developed countries. This was mentioned for Japan (83) and Korea (80). For New Zealand authors argued that inpatient psychiatric bed capacity and availability generates utilization and coercive treatments (57). Economic incentives for inadequately long inpatient bed use have been observed in Japan (116). In the UK, it was suggested that bed reductions lead to better use and development of existing community care, along with reduced reliance on such beds (100). Lastly, for the US it is suggested that hospital bed numbers should be reduced to serve the most severely ill patients (90).

Opinions were divided on themes, such as the costeffectiveness of psychiatric bed reductions. On the one hand, the lower cost of community services compared to inpatient care allows for greater concentration of inpatient resources on the most severely ill. However, in 1998 an US-American study showed increased costs in the entire health system after bed reductions due to a higher use of acute care in general hospitals as a result of severely mentally ill patients queuing up in these facilities while waiting for transfers to intermediate care in the community (115). In regard to quality of care opinions were also divided. Lack of beds can lead to reduced quality of inpatient treatments and overcrowding. However, hospitals in the US have shown to maintain or increase their quality of care by removing beds while maintaining the staff (82).

Our study shows that an empirical approach to argue for trends of psychiatric bed numbers was most frequent, while the normative approach was least frequently used with no differences between HICs and LMICs. It has been argued that need of psychiatric beds may vary between regions (131), and even changes between seasons (132). The provision of psychiatric beds has to respond to local requirements and conditions (127). The arguments presented here may help to tailor policies after evaluation of fit and contextdependent applicability. One-dimensional models that simply focus on the numbers to address possible bed shortages may need to consider incorporating more complex aspects of the system, including costs, quality and pathways of care (133). At the level of catchment areas, the need of beds has to be evaluated assuring a continuum of care with quality treatment before, during and after an acute episode of mental illness (134).

\section{Strengths and Limitations}

To our knowledge this is the first systematic review of expert arguments on psychiatric bed numbers. We provide evidence and examined arguments from 25 countries worldwide, including 14 LMICs. This research has several limitations. The arguments in the present review were not necessarily identical to the main objective of the respective studies but were extracted from the discussions during our screening process. Secondly, the usage of variable nomenclatures referring to psychiatric beds is a further limitation. Thirdly, study quality was not assessed. Another limitation was that this review did not assess the opinions of patients, caregivers, families and other members of the community.

\section{Conclusion}

Several implications arise from our findings. First, there are relatively few arguments that are repeated in the literature and should be considered by discussants on the required number of psychiatric beds. This synthesis of arguments can help to focus future debate and to guide policymakers who need to define targets for the number of psychiatric beds in specific countries and catchment areas. Secondly, further research is needed to guide which arguments are best suited for specific contexts. Thirdly, there is need to establish targets for more specific populations (e.g., juvenile, older adult, forensic, acute and long-stay populations) and for specific types of facilities (public, private, mental or general hospital and residential). The low number of publications from LMICs highlights the need for further evidence from these countries, especially from regions underrepresented 
in research, such as Central and East Asia, Africa and Central America.

\section{DATA AVAILABILITY STATEMENT}

The original contributions presented in the study are included in the article/supplementary material, further inquiries can be directed to the corresponding author/s.

\section{AUTHOR CONTRIBUTIONS}

Literature screening was conducted by SD, ER, and MS. Data were extracted independently by SD, ER, and MS. SD and AM performed the data analysis. ER and MS reviewed it. SD wrote

\section{REFERENCES}

1. Goodwin S. Comparative Mental Health Policy: From Institutional to Community Care. Manchester: Sage (1997).

2. Fakhoury W, Priebe S. The process of deinstitutionalization: an international overview. Curr Opin Psychiatry. (2002) 15:187-92. doi: 10.1097/00001504-200203000-00011

3. Priebe S, Badesconyi A, Fioritti A, Hansson L, Kilian R, Torres-Gonzales F, et al. Reinstitutionalisation in mental health care: comparison of data on service provision from six European countries. BMJ. (2005) 330:123-6. doi: 10.1136/bmj.38296.611215.AE

4. Trieman N, Leff J. Long-term outcome of long-stay psychiatric inpatients considered unsuitable to live in the community: TAPS Project 44. Br J Psychiatry. (2002) 181:428-32. doi: 10.1192/bjp.181. 5.428

5. Bastiampillai TJ, Bidargaddi NP, Dhillon RS, Schrader GD, Strobel JE, Galley PJ. Implications of bed reduction in an acute psychiatric service. Med J Aust. (2010) 193:383-6. doi: 10.5694/j.1326-5377.2010.tb03963.x

6. Shumway M, Alvidrez J, Leary M, Sherwood D, Woodard E, Lee EK, et al. Impact of capacity reductions in acute public-sector inpatient psychiatric services. Psychiatr Serv. (2012) 63:135-41. doi: 10.1176/appi.ps.2010 00145

7. Tyrer P. Has the closure of psychiatric beds gone too far? Yes. BMJ. (2011) 343:d7457. doi: 10.1136/bmj.d7457

8. Mundt AP, Chow WS, Arduino M, Barrionuevo H, Fritsch R, Girala N, et al. Psychiatric hospital beds and prison populations in South America since 1990: does the Penrose hypothesis apply? JAMA Psychiatry. (2015) 72:112-8. doi: 10.1001/jamapsychiatry.2014.2433

9. Ose SO, Kalseth J, Ådnanes M, Tveit T, Lilleeng SE. Unplanned admissions to inpatient psychiatric treatment and services received prior to admission. Health Policy. (2018) 122:359-66. doi: 10.1016/j.healthpol.2017.12.006

10. Guaiana G, Bastiampillai T, Allison S, O’Reilly R. Basaglia’s impact. Lancet. (2019) 6:94-5. doi: 10.1016/S2215-0366(18)30506-6

11. World Health Organization. Mental Health ATLAS 2017. (2018). Available online at: https://apps.who.int/iris/handle/10665/272735 (accessed May 20, 2021).

12. The World Bank. World Bank Country and Lending Groups. (2020). Available online at: https://datahelpdesk.worldbank.org/knowledgebase/ articles/906519-world-bank-country-and-lending-groups

(accessed December 14, 2020)

13. Oecd Health Statistics 2020. Definitions, Sources and Methods: All Psychiatric Care Beds in Hospitals. OECD Health Statistics (2020). Available online at: https://www.oecd.org/health/health-data.htm (accessed December 19, 2020).

14. Lelliott $\mathrm{P}$, Wing J. A national audit of new long-stay psychiatric patients. II: Impact on services. Br J Psychiatry. (1994) 165:170-8. doi: 10.1192/bjp.165.2.170 the manuscript. AM and SP revised and corrected it along the process. All authors contributed to the article and approved the submitted version.

\section{FUNDING}

This systematic review was funded by the Agencia Nacional de Investigación y Desarrollo in Chile, grant scheme FONDECYT Regular, Grant No. 1190613.

\section{ACKNOWLEDGMENTS}

We acknowledge support from the German Research Foundation (DFG) and the Open Access Publication Fund of Charité Universitätsmedizin Berlin.

15. Daly A, Walsh D. An audit of new long-stay patients in Irish psychiatric inpatient services. Ir J Psychol Med. (2009) 26:134-9. doi: 10.1017/S0790966700000446

16. Pirkis J, Harris M, Buckingham W, Whiteford H, Townsend-White C. International planning directions for provision of mental health services. Administ Policy Mental Health Mental Health Serv Res. (2007) 34:377-87. doi: 10.1007/s10488-007-0116-0

17. Pinals DA, Fuller DA. Beyond Beds: The Vital Role of a Full Continuum of Psychiatric Care. National Association of State Mental Health Program Directors \& Treatment Advocacy Center. Arlington, VA (2017).

18. O'Reilly R, Allison S, Bastiampiallai T. Observed outcomes: an approach to calculate the optimum number of psychiatric beds. Adm Policy Ment Health. (2019) 46:507-17. doi: 10.1007/s10488-018-00917-8

19. Harris MG, Buckingham WJ, Pirkis J, Groves A, Whiteford H. Planning estimates for the provision of core mental health services in Queensland 2007 to 2017. Aust N Z J Psychiatry. (2012) 46:982-94. doi: $10.1177 / 0004867412452942$

20. Muller C. The future of the psychiatric institution: Utopia or reality? [The future of the psychiatric institution: Utopia or reality?]. Soc Psychiatry. (1973) 8:185-91.

21. Gordon A. Psychiatric bed levels. Position Paper Canad Psychiatric Assoc Bull. (1997) 29:1-4.

22. Fuller E, Entsminger K, Geller J, Stanley J, Jaffe DJ. The Shortage of Public Hospital Beds for Mentally Ill Persons - A Report of the Treatment Advocacy Center (ed.) Treatment Advocacy Center. Arlington, VA (2008).

23. Braun V, Clarke V. Using thematic analysis in psychology. Qualit Res Psychol. (2006) 3:77-101. doi: 10.1191/1478088706qp063oa

24. Lachal J, Revah-Levy A, Orri M, Moro MR. Metasynthesis: an original method to synthesize qualitative literature in psychiatry. Front Psychiatry. (2017) 8:269. doi: 10.3389/fpsyt.2017.00269

25. Allison S, Bastiampillai T. Mental health services reach the tipping point in Australian acute hospitals. Med J Austr. (2015) 203:432-4. doi: $10.5694 / \mathrm{mja} 15.00782$

26. Baia Medeiros DT, Hahn-Goldberg S, Aleman DM, O'Connor E. Planning capacity for mental health and addiction services in the emergency department: a discrete-event simulation approach. J Healthc Eng. (2019) 2019:8973515. doi: 10.1155/2019/8973515

27. Bloom JD. Psychiatric boarding in Washington State and the inadequacy of mental health resources. J Am Acad Psychiatry Law. (2015) 43:218-22.

28. Claudius I. Comparison of youth suicide with involuntary hold criteria and inpatient T capacity. Mental Health Prevent. (2019) 15:200172. doi: 10.1016/j.mhp.2019.200172

29. Davie M. It's chaos out here. Aust N Z J Psychiatry. (2019) 53:87-8. doi: $10.1177 / 0004867418778772$

30. Dhillon R. A failed experiment: Substitution of acute inpatient beds by subacute community residential beds in South Australia. Aust N Z J Psychiatry. (2015) 49:579-80. doi: 10.1177/0004867415573056 
31. Duthie P. In-patient adolescent services. Psychiatric Bull. (2001) 25:360. doi: $10.1192 / \mathrm{pb} .25 .9 .360$

32. Early DF, Nicholas M. The developing scene: ten-year review of a psychiatric hospital population. Br Med J. (1971) 4:793-5. doi: 10.1136/bmj.4.5790.793

33. Early DF, Nicholas M. Dissolution of the mental hospital: Fifteen years on. Br J Psychiatry. (1977) 130:117-22. doi: 10.1192/bjp.130.2.117

34. Elpers JR, Crowell A. How many beds? An overview of resource planning. Hosp Community Psychiatry. (1982) 33:755-61. doi: 10.1176/ps.33.9.755

35. Fagundes Junior HM, Desviat M, Silva PR. Psychiatric Reform in Rio de Janeiro: the current situation and future perspectives. Cien Saude Colet. (2016) 21:1449-60. doi: 10.1590/1413-81232015215.00872016

36. Flannigan CB, Glover GR, Feeney ST, Wing JK, Bebbington PE, Lewis SW. Inner London collaborative audit of admissions in two health districts: I Introduction, methods and preliminary findings. Br J Psychiatry. (1994) 165:734-42. doi: 10.1192/bjp.165.6.734

37. Ford R, Minghella E, Cahlmers C, Hoult J, Raftery J, Muijen M. Cost consequences of home-based and in-patient-based acute psychiatric treatment: Results of an implementation study. J Mental Health. (2001) 10:467-76. doi: 10.1080/09638230120041236

38. Friebel R, Fisher R, Deeny SR, Gardner T, Molloy A, Steventon A. The implications of high bed occupancy rates on readmission rates in England: A longitudinal study. Health Policy. (2019) 123:765-72. doi: 10.1016/j.healthpol.2019.06.006

39. Fulop NJ, Koffman J, Carson S, Robinson A, Pashley D, Coleman K. Use of acute psychiatric beds: A point prevalence survey in North and South Thames regions. J Public Health Med. (1996) 18:207-16. doi: 10.1093/oxfordjournals.pubmed.a024481

40. Harris DM. An elaboration of the relationship between general hospital bed supply and general hospital utilization. J Health Soc Behav. (1975) 16:163-72. doi: $10.2307 / 2137157$

41. Hatta $K$, Nakamura $H$, Usui C, Kurosawa H. Utility and sufficiency of psychiatric inpatient units in general hospitals: A crosssectional study in Tokyo. Psychiatry Clin Neurosci. (2010) 64:642-4. doi: $10.1111 / j .1440-1819.2010 .02139 . x$

42. Jones R. Optimum bed occupancy in psychiatric hospitals. Psychiatry OnLine. (2013) 552:1-9.

43. Kalucy R, Thomas L, King D. Changing demand for mental health services in the emergency department of a public hospital. Aust N Z J Psychiatry. (2005) 39:74-80. doi: 10.1080/j.1440-1614.2005.01512.x

44. Kelly CB. An audit of acute psychiatric admission bed occupancy in Northern Ireland. Ulster Med J. (1998) 67:44-8.

45. Keown P, Tacchi MJ, Niemiec S, Hughes J. Changes to mental healthcare for working age adults: impact of a crisis team and an assertive outreach team. Psychiatric Bull. (2007) 31:288-92. doi: 10.1192/pb.bp.106.012054

46. La EM, Lich KH, Wells R, Ellis AR, Swartz MS, Zhu R, et al. Increasing access to state psychiatric hospital beds: exploring supply-side solutions. Psychiatr Serv. (2016) 67:523-8. doi: 10.1176/appi.ps.201400570

47. Lamb HR, Weinberger LE. Meeting the needs of those persons with serious mental illness who are most likely to become criminalized. J Am Acad Psychiatry Law Online. (2011) 39:549-54.

48. Laugharne R, Branch M, Mitchell A, Parkin L, Confue P, Shankar R, et al. What happens when $55 \%$ of acute psychiatric beds are closed in six days: an unexpected naturalistic observational study. JRSM Open. (2016) 7:1-8. doi: $10.1177 / 2054270416649280$

49. Lee EH-M, So H-C, Chen EY-H. Admission rates and psychiatric beds in Hong Kong, 1999-2014: A population-based study. Psychiatr Serv. (2016) 67:579. doi: 10.1176/appi.ps.201600026

50. Lelliott P. Meeting the accommodation needs of the most severely mentally ill. J Interprofessional Care. (1996) 10:241-7. doi: 10.3109/13561829609034111

51. Lelliott P. Acute inpatient psychiatry in England: An old problem and a new priority. Epidemiol Psichiatr Soc. (2006) 15:91-4. doi: 10.1017/S1121189X00004267

52. Lippert SC, Jain N, Nesper A, Fahimi J, Pirrotta E, Wang NE. Waiting for care: Differences in emergency department length of stay and disposition between medical and psychiatric patients. Ann Emerg Med. (2016) 68:S97. doi: 10.1016/j.annemergmed.2016.08.263
53. Loch AA, Gattaz WF, Rossler W. Mental healthcare in South America with a focus on Brazil: past, present, and future. Curr Opin Psychiatry. (2016) 29:264-9. doi: 10.1097/YCO.0000000000000259

54. Long R. Acute psychiatry bed shortage contributing to iatrogenic deterioration in general hospital wards. Aust N Z J Psychiatry. (2015) 49:391. doi: 10.1177/0004867414561530

55. Lund C, Flisher AJ. Norms for mental health services in South Africa. Soc Psychiatry Psychiatr Epidemiol. (2006) 41:587-94. doi: 10.1007/s00127-006-0057-z

56. Macdonald J, Jackson T, Ellis PM. Did Wellington have too many acute psychiatric beds in 1996? New Zealand Med J. (1999) 112:275-6.

57. Malcolm L. Bed availability as a significant influence on rates of committal to New Zealand's psychiatric hospitals. N Z Med J. (1989) 102:8-9.

58. Morris J, Lora A, Mcbain R, Saxena S. Global mental health resources and services: a WHO survey of 184 countries. Mental Health Public Health Issue. (2012) 34:91671. doi: 10.1007/BF03391671

59. Munk-Jørgensen P. Has deinstitutionalization gone too far? Eur Arch Psychiatry Clin Neurosci. (1999) 249:136-43. doi: 10.1007/s004060050078

60. Nicks BA, Manthey DM. The impact of psychiatric patient boarding in emergency departments. Emerg Med Int. (2012) 2012:1-5. doi: $10.1155 / 2012 / 360308$

61. Mundt AP, Konrad N. Institutionalization, deinstitutionalization, and the Penrose hypothesis. Adv Psychiatry. (2019) 2019:187-96. doi: 10.1007/978-3-319-70554-5_12

62. Niehaus DJH, Koen L, Galal U, Dhansay K, Oosthuizen PP, Emsley RA, et al. Crisis discharges and readmission risk in acute psychiatric male inpatients. BMC Psychiatry. (2008) 8:44. doi: 10.1186/1471-244X-8-44

63. Nordstrom K, Berlin JS, Nash SS, Shah SB, Schmelzer NA, Worley LLM. Boarding of mentally ill patients in emergency departments: American Psychiatric association resource document. West J Emerg Med. (2019) 20:690-5. doi: 10.5811/westjem.2019.6.42422

64. O'Doherty M. Acute psychiatric services: An appraisal of a major change in service delivery within one catchment area. Ir J Psychol Med. (1998) 15:84-7. doi: $10.1017 /$ S0790966700003736

65. O'Reilly RL, Chamberlaine $\mathrm{CH}$. Criteria for admission to psychiatric units: Have we raised the bar too high? Canad J Psychiatry. (2000) 45:392.

66. O’Neil AM, Sadosty AT, Pasupathy KS, Russi C, Lohse CM, Campbell RL. Hours and miles: patient and health system implications of transfer for psychiatric bed capacity. West J Emerg Med. (2016) 17:783. doi: 10.5811/westjem.2016.9.30443

67. Parker S, Siskind D, Harris M. Community based residential mental health services: What do we need to know? Aust N Z J Psychiatry. (2015) 49:86-7. doi: $10.1177 / 0004867414557163$

68. Pelzang R. The pattern of psychiatric admissions in a referral hospital, Bhutan. WHO South-East Asia J Public Health. (2012) 1:52. doi: 10.4103/2224-3151.206914

69. Powell RB, Hollander D, Tobiansky RI. Crisis in admission beds. $\mathrm{Br} J$ Psychiatry. (1995) 167:765-9. doi: 10.1192/bjp.167.6.765

70. Prins SJ. Does transinstitutionalization explain the overrepresentation of people with serious mental illnesses in the criminal justice system? Community Ment Health J. (2011) 47:716-22. doi: 10.1007/s10597-011-9420-y

71. Saraceno B, Gater R, Rahman A, Saeed K, Eaton J, Ivbijaro G, et al. Reorganization of mental health services: from institutional to communitybased models of care. Eastern Mediter Health J. (2015) 21:477-85. doi: $10.26719 / 2015.21 .7 .477$

72. Thomas LE. Psychiatric emergencies. Child Adolesc Psychiatr Clin N Am. (2003) 12:xiii-xv. doi: 10.1016/S1056-4993(03)00069-5

73. Tyrer P, Sharfstein S, O'Reilly R, Allison S, Bastiampillai $T$. Psychiatric hospital beds: an Orwellian crisis. Lancet. (2017) 389:363. doi: 10.1016/S0140-6736(17)30149-6

74. Akpalu B, Lund C, Doku V, Ofori-Atta A, Osei A, Ae-Ngibise K, et al. Scaling up community-based services and improving quality of care in the state psychiatric hospitals: the way forward for Ghana. Afr J Psychiatry. (2010) 13:109-15. doi: 10.4314/ajpsy.v13i2.54356

75. Tim P. Improving inpatient mental health care in the UK. J R Soc Med. (2013) 382:660. doi: 10.1016/S0140-6736(13)61761-4 
76. Barnett BS, Kusunzi V, Magola L, Borba CPC, Udedi M, Kulisewa K, et al. Factors associated with long length of stay in an inpatient psychiatric unit in Lilongwe, Malawi. Soc Psychiatry Psychiatr Epidemiol. (2019) 54:235-42. doi: 10.1007/s00127-018-1611-1

77. Giel R. Care of chronic mental patients in The Netherlands. Soc Psychiatry. (1986) 21:25-32. doi: 10.1007/BF00585319

78. Hailey AM. Long-stay psychiatric inpatients: a study based on the Camberwell Register. Psychol Med. (1971) 1:128-42. doi: 10.1017/S0033291700000052

79. Holloway F, Wykes T, Petch E, Lewis-Cole K. The new long stay in an inner city service: A tale of two cohorts. Int J Soc Psychiatry. (1999) 45:93-103. doi: 10.1177/002076409904500202

80. Kim AM. Why do psychiatric patients in Korea stay longer in hospital? Int J Ment Health Syst. (2017) 11:2. doi: 10.1186/s13033-016-0110-6

81. Lesage AD, Tansella M. Comprehensive community care without long stay beds in mental hospitals: Trends from an Italian good practice area. Canad J Psychiatry. (1993) 38:187-94. doi: 10.1177/070674379303800307

82. Madianos MG. Deinstitutionalization and the closure of public mental hospitals: the pragmatics of psychiatric reform in Greece. Int J Ment Health. (2002) 31:66-75. doi: 10.1080/00207411.2002.11449566

83. Okayama T, Usuda K, Okazaki E, Yamanouchi Y. Number of longterm inpatients in Japanese psychiatric care beds: trend analysis from the patient survey and the 630 survey. BMC Psychiatry. (2020) 20:2927. doi: 10.1186/s12888-020-02927-z

84. Sisti DA, Segal AG, Emanuel EJ. Improving long-term psychiatric care: bring back the asylum. JAMA. (2015) 313:243-4. doi: 10.1001/jama.2014.16088

85. Allison S, Bastiampillai T, Fuller DA. Should the Government change the Mental Health Act or fund more psychiatric beds? Lancet. (2017) 4:585-6. doi: 10.1016/S2215-0366(17)30290-0

86. Bowersox NW, Szymanski BJ, Mccarthy JF. Associations between psychiatric inpatient bed supply and the prevalence of serious mental illness in Veterans Affairs nursing homes. Am J Public Health. (2013) 103:1325-31. doi: 10.2105/AJPH.2012.300783

87. Dazzan P, Barbui C. From the 16th to the 21st Century: how we approach mental health problems and where do we go next? Epidemiol Psychiatric Sci. (2015) 24:365-7. doi: 10.1017/S204579601500061X

88. De Vetten M, Shields-Zeeman L, Petrea I, Klazinga N. Assessing the need for a mental health services reform in Moldova: a situation analysis. Int J Ment Health Syst. (2019) 13:45. doi: 10.1186/s13033-019-0292-9

89. Fioritti A, Lo Russo L, Melega V. Reform said or done? The case of EmiliaRomagna within the Italian psychiatric context. Am J Psychiatry. (1997) 154:94-8. doi: 10.1176/ajp.154.1.94

90. Fisher WH, Simon L, Geller J, Penk WE, Irvin EA, White CL. Case mix in the "downsizing" state hospital. Psychiatr Serv. (1996) 47:255-62. doi: $10.1176 /$ ps.47.3.255

91. Forchuk C, Russell G, Kingston-Macclure S, Turner K, Dill S. From psychiatric ward to the streets and shelters. J Psychiatr Ment Health Nurs. (2006) 13:301-8. doi: 10.1111/j.1365-2850.2006.00954.x

92. Forrester A, Exworthy T, Chao O, Slade K, Parrott J. Influencing the care pathway for prisoners with acute mental illness. Crim Behav Ment Health. (2013) 23:217-26. doi: 10.1002/cbm.1870

93. Geller JL, Biebel K. The premature demise of public child and adolescent inpatient psychiatric beds: Part II: challenges and implications. Psychiatr Q. (2006) 77:273-91. doi: 10.1007/s11126-006-9013-z

94. Geng F, Jiang F, Rakofsky J, Liu T, Liu Y, Liu H, et al. Psychiatric inpatient beds for youths in China: data from a nation-wide survey. BMC Psychiatry. (2020) 20:398. doi: 10.1186/s12888-020-02802-x

95. Goldman RL, Keller TW. The effectiveness of planning for community mental health center inpatient units. Community Ment Health J. (1978) 14:100-9. doi: 10.1007/BF00778828

96. Hartvig P, Kjelsberg E. Penrose's law revisited: the relationship between mental institution beds, prison population and crime rate. Nord J Psychiatry. (2009) 63:51-56. doi: 10.1080/08039480802298697

97. Hollander D, Powell R, Tobiansky R. Bed occupancy in psychiatric units in Greater London is 113\% [1]. Br Med J. (1996) 313:166. doi: $10.1136 /$ bmj.313.7050.166

98. Hume PB, Rudin E. Psychiatric inpatient services in general hospitals. Calif Med. (1960) 93:200-7.
99. Jeppesen RM, Christensen T, Vestergaard $\mathrm{CH}$. Changes in the utilization of psychiatric hospital facilities in Denmark by patients diagnosed with schizophrenia from 1970 through 2012. Acta Psychiatr Scand. (2016) 133:419-25. doi: 10.1111/acps.12549

100. Johnson S. Has the closure of psychiatric beds gone too far? No. BMJ. (2011) 343:d7410. doi: 10.1136/bmj.d7410

101. Kaltiala-Heino R, Laippala P, Joukamaa M. Has the attempt to reduce psychiatric inpatient treatment been successful in Finland? Eur Psychiatry (2001) 16:215-21. doi: 10.1016/S0924-9338(01)00567-3

102. Keown P, Mckenna D, Murphy H, Mckinnon I. Offenders with mental disorders in prison and the courts: links to rates of civil detentions and the number of psychiatric beds in England - longitudinal data from 1984 to 2016 BJPsych Open. (2019) 5:1-7. doi: 10.1192/bjo.2019.73

103. Kigozi F, Ssebunnya J, Kizza D, Cooper S, Ndyanabangi S. An overview of Uganda's mental health care system: Results from an assessment using the World Health Organization's Assessment Instrument for Mental Health Systems (WHOAIMS). Int J Mental Health Syst. (2010) 4:1. doi: 10.1186/1752-4458-4-1

104. Kilsztajn S, Lopes ES, Lima LZ, Rocha PAF, Carmo MSN. Hospital beds and mental health reform in Brazil. Leitos Hospit Reforma Psiquiatrica Brasil. (2008) 24:2354-62. doi: 10.1590/S0102-311X2008001000016

105. Lamb HR. Does deinstitutionalization cause criminalization?: The penrose hypothesis. JAMA Psychiatry. (2015) 72:105-6. doi: 10.1001/jamapsychiatry.2014.2444

106. Lawrence RE, Copas JB, Cooper PW. Community care: does it reduce the need for psychiatric beds? A comparison of two different styles of service in three hospitals. Br J Psychiatry. (1991) 159:334-40. doi: 10.1192/bjp.159.3.334

107. Lelliott $\mathrm{P}$, Audini B. Trends in the use of part ii of the mental health act 1983 in seven English local authority areas. Br J Psychiatry. (2003) 182:68-70 doi: 10.1192/bjp.182.1.68

108. Macdonald LD. Community psychiatric care in Italy: The Rome example. Int J Soc Psychiatry. (1989) 35:71-80. doi: 10.1177/002076408903500108

109. Munk-Jørgensen P, Mortensen PB. Is schizophrenia really on the decrease? Eur Arch Psychiatry Clin Neurosci. (1993) 242:244-7. doi: $10.1007 / \mathrm{BF} 02189970$

110. Nilsson LL, Logdberg B. Dead and forgotten-postmortem time before discovery as indicator of social isolation and inadequate mental healthcare in schizophrenia. Schizophr Res. (2008) 102:337-9. doi: 10.1016/j.schres.2008.03.011

111. Nome S, Holsten F. A prospective longitudinal study of utilization of a psychiatric hospital in Hordaland County, Norway, from 1985 to 2003. Nord J Psychiatry. (2011) 65:117-24. doi: 10.3109/08039488.2010.504991

112. Nordentoft M, Knudsen HC, Jessen-Petersen B, Krasnik A, Saelan H, Treufeldt P, et al. CCPP-Copenhagen Community Psychiatric Project. Implementation of community mental health centres in Copenhagen: effects of service utilization, social integration, quality of life and positive and negative symptoms. Soc Psychiatry Psychiatr Epidemiol. (1996) 31:336-44. doi: 10.1007/BF00783422

113. O’Neill C, Sinclair H, Kelly A, Kennedy HG. Interaction of forensic and general psychiatric services in Ireland: learning the lessons or repeating the mistakes. Ir J Psychol Med. (2002) 19:48-54. doi: 10.1017/S0790966700006959

114. Roberts M, Mogan C, Asare JB. An overview of Ghana's mental health system: results from an assessment using the World Health Organization's Assessment Instrument for Mental Health Systems (WHO-AIMS). Int J Mental Health Syst. (2014) 8:16. doi: 10.1186/1752-4458-8-16

115. Rothbard AB, Schinnar AP, Hadley TP, Foley KA, Kuno E. Cost comparison of state hospital and community-based care for seriously mentally ill adults. Am J Psychiatry. (1998) 155:523-9. doi: 10.1176/ajp.155.4.523

116. Sasaki H. The role of private sector psychiatry in the Japanese mental health system. Int J Ment Health. (2012) 41:61-71. doi: 10.2753/IMH0020-7411410206

117. Someya T, Suzuki Y, Sham PC, Tang SW. Forecasting the number of inpatients with schizophrenia. Psychiatry Clin Neurosci. (2004) 58:573-8. doi: 10.1111/j.1440-1819.2004.01302.x

118. Švab V, Groleger U, Ziherl S. The development of psychiatric reform in Slovenia. World Psychiatry. (2006) 5:56 
119. Fuller ET, Fuller DA, Geller J, Jacobs C, Ragosta K. No Room at the Inn: Trends and Consequences of Closing Public Psychiatric Hospitals. Arlington, VA: Treatment Advocacy Center (2012).

120. Trieman N, Leff J. The TAPS project. 36: the most difficult to place long-stay psychiatric in-patients. Br J Psychiatry. (1996) 169:289-92. doi: 10.1192/bjp.169.3.289

121. Weller MPI, Weller BGA. Crime and mental illness. Med Sci Law. (1988) 28:38-46. doi: 10.1177/002580248802800111

122. Worrall A, O'Herlihy A. Psychiatrists' views of in-patient child and adolescent mental health services: A survey of members of the child and adolescent faculty of the College. Psychiatric Bull. (2001) 25:219-22. doi: $10.1192 / \mathrm{pb} .25 .6 .219$

123. Yoon J, Bruckner TA. Does deinstitutionalization increase suicide? Health Serv Res. (2009) 44:1385-405. doi: 10.1111/j.1475-6773.2009.00986.x

124. Yoon J, Domino ME, Norton EC, Cuddeback GS, Morrissey JP. The impact of changes in psychiatric bed supply on jail use by persons with severe mental illness. J Mental Health Policy Econ. (2013) 16:81-92.

125. Allison S, Bastiampillai T, Castle D. Victoria's low availability of public psychiatric beds and the impact on patients, carers and staff. Aust N Z J Psychiatry. (2018) 52:91-2. doi: 10.1177/0004867417721019

126. Bagcchi S. Mentally ill patient chained to tree to highlight shortage of psychiatric beds in India. BMJ. (2013) 347:f5604. doi: 10.1136/bmj.f5604

127. Rowland LA, Zeelan J, Waismann LC. Patterns of service for the long-term mentally ill in Europe. Br J Clin Psychol. (1992) 31:405-17. doi: 10.1111/j.2044-8260.1992.tb01015.x

128. Allison S, Bastiampillai T, Licinio J, Fuller DA, Bidargaddi N, Sharfstein SS. When should governments increase the supply of psychiatric beds? Mol Psychiatry. (2018) 23:796-800. doi: 10.1038/mp.2017.139

129. Andrews G. Private and public psychiatry: a comparison of two health care systems. Am J Psychiatry. (1989) 146:881-6. doi: 10.1176/ajp.146.7.881

130. Yadawad V, Ganjekar S, Thippeswamy H, Chandra PS, Desai G. Shortterm outcome of mothers with severe mental illness admitted to a mother baby unit. Indian J Psychiatry. (2021) 63:245-9. doi: 10.4103/ psychiatry.IndianJPsychiatry_1005_20

131. Hirsch SR. Planning for bed needs and resource requirements in acute psychiatry: key points from the report of the Royal college of psychiatrists working party on psychiatric beds and resources, 1986. Bull $R$ College Psychiatrists. (1987) 11:398-407. doi: 10.1192/pb.11.12.398

132. Jones RP. Myths of ideal hospital size. Med J Aust. (2010) 193:298-300. doi: 10.5694/j.1326-5377.2010.tb03912.x

133. Middleton H. Bed numbers and acute in-patient care. Psychiatric Bull. (2007) 31:233. doi: $10.1192 / \mathrm{pb} .31 .5 .233 \mathrm{a}$

134. Pinals DA, Fuller DA. The vital role of a full continuum of psychiatric care beyond beds. Psychiatr Serv. (2020) 71:713-21. doi: 10.1176/appi.ps.201900516

Conflict of Interest: The authors declare that the research was conducted in the absence of any commercial or financial relationships that could be construed as a potential conflict of interest.

Publisher's Note: All claims expressed in this article are solely those of the authors and do not necessarily represent those of their affiliated organizations, or those of the publisher, the editors and the reviewers. Any product that may be evaluated in this article, or claim that may be made by its manufacturer, is not guaranteed or endorsed by the publisher.

Copyright (C) 2021 Mundt, Delhey Langerfeldt, Rozas Serri, Siebenförcher and Priebe. This is an open-access article distributed under the terms of the Creative Commons Attribution License (CC BY). The use, distribution or reproduction in other forums is permitted, provided the original author(s) and the copyright owner(s) are credited and that the original publication in this journal is cited, in accordance with accepted academic practice. No use, distribution or reproduction is permitted which does not comply with these terms. 